Piecewise linear loop quantum gravity

This article has been downloaded from IOPscience. Please scroll down to see the full text article.

2010 Class. Quantum Grav. 27035003

(http://iopscience.iop.org/0264-9381/27/3/035003)

View the table of contents for this issue, or go to the journal homepage for more

Download details:

IP Address: 194.94.224.254

The article was downloaded on 30/08/2012 at $11: 10$

Please note that terms and conditions apply. 


\title{
Piecewise linear loop quantum gravity
}

\author{
Jonathan Engle ${ }^{1,2,3}$ \\ ${ }^{1}$ MPI für Gravitationsphysik (Albert-Einstein-Institut), Am Mühlenberg 1, D-14476 Potsdam, \\ Germany \\ ${ }^{2} \mathrm{CPT}^{4}$, CNRS Case 907, Université de la Méditerranée, D-13288 Marseille, France \\ ${ }^{3}$ Institut f. Th. Physik III, Universität Erlangen-Nürnberg, Staudtstraße 7, D-91058 Erlangen, \\ Germany
}

Received 10 July 2009, in final form 1 December 2009

Published 12 January 2010

Online at stacks.iop.org/CQG/27/035003

\begin{abstract}
We define a modification of loop quantum gravity (LQG) in which graphs are required to consist of piecewise linear edges, which we call piecewise linear LQG (plLQG). At the diffeomorphism-invariant level, we prove that plLQG is equivalent to standard LQG, as long as one chooses the class of diffeomorphisms appropriately. That is, we exhibit a unitary map between the diffeomorphism-invariant Hilbert spaces that maps physically equivalent operators into each other. In addition, using the same ideas as in standard LQG, one can define a Hamiltonian and master constraint in plLQG, and the unitary map between pILQG and LQG then provides an exact isomorphism of dynamics in the two frameworks. Furthermore, loop quantum cosmology (LQC) can be exactly embedded into plLQG. This allows a prior program of the author to embed LQC into LQG at the dynamical level to proceed. In particular, this allows a formal expression for a physically motivated embedding of LQC into LQG at the diffeomorphism-invariant level to be given.
\end{abstract}

PACS numbers: $04.60 . \mathrm{Ds}, 04.60 . \mathrm{Kz}, 04.60 . \mathrm{Pp}, 02.40 . \mathrm{Re}, 02.40 . \mathrm{Sf}$

\section{Introduction}

Loop quantum gravity (LQG) [1-3] is a minimalistic, background-independent approach to quantum gravity. However, in the construction of the theory, technical choices have to be made, especially in the kinematics of the theory. One can then ask: might some of these technical choices not matter once the constraints are solved? In this paper, we show that in particular the choice of the piecewise analytic category is not essential: it can even be replaced with something as simple as the piecewise linear category, and the resulting theory is the same at the diffeomorphism-invariant level. The diffeomorphism-invariant Hilbert spaces of the

4 Unité mixte de recherche (UMR 6207) du CNRS et des Universités de Provence (Aix-Marseille I), de la Meditarranée (Aix-Marseille II) et du Sud (Toulon-Var); laboratoire affilié à la FRUMAM (FR 2291). 
two theories are naturally isomorphic, and the dynamics are exactly the same. Furthermore, a very large algebra of the diffeomorphism-invariant operators are also seen to be the same.

We call this modification of LQG 'piecewise linear loop quantum gravity' (plLQG).

What are the consequences of this? First, plLQG can be used as a 'trick' to circumvent an obstruction to the program of $[4,5]$, allowing the program to proceed. The program $[4,5]$ lays out a strategy for embedding loop quantum cosmology - the symmetry reduced version of loop quantum gravity-into LQG. Prior to the present work, a seemingly technical detail hindered the program: as often suspected, and finally proven in [6], the restriction, to the homogeneous isotropic sector, of the basic algebra of configuration observables in LQG (the cylindrical functions) is not equal to the basic configuration algebra in LQC (the almost periodic functions). We refer the reader to section 5 of the present paper for an explanation of why this is a hindrance. Piecewise linear LQG, by contrast, does not have this hindrance. This, combined with the fact that plLQG is completely equivalent to standard LQG at the diffeomorphism-invariant level, finally allows the systematic program of [4, 5] to move forward, leading to a formal expression for an embedding of LQC into LQG at the diffeomorphism-invariant level. A second possible use of the piecewise linear framework is that it may allow a closer relation to spinfoams [7, 8], which also use the piecewise linear category to define the kinematics [8].

It should be noted that, in order to achieve isomorphism with LQG, one must use in LQG a class of generalized diffeomorphisms allowing non-differentiability on lower dimensional surfaces, such as that systematically motivated in [9], and advocated in [14, 22]. This in turn forces one to use the Rovelli-Smolin ordering of the volume operator [1, 2, 10, 12] instead of the Ashtekar-Lewandowski ordering $[1,2,11,12]$ when defining the Hamiltonian and master constraints via the usual construction $[15,16]$.

After this work was completed, it was pointed out to the author that the kinematics of piecewise linear LQG as presented here, and the choice of generalized diffeomorphisms, had already been proposed as a model by Zapata in [13]. In [13], one was not interested in plLQG as such, and so did not develop it beyond kinematics. This paper goes further, in rigorously constructing the rigging map for the diffeomorphism constraint, constructing Hamiltonian and master constraint operators and showing equivalence with the piecewise analytic framework at the diffeomorphism-invariant level including dynamics. Of course the embedding of LQC into plLQG is also new. On the other hand, [13] presents features of the kinematics of plLQG not presented here. For example, [13] introduces the piecewise linear analog $\overline{\mathcal{A}}_{\mathrm{PL}}$ of the generalized connections, and constructs the piecewise linear analog of the Ashtekar-Lewandowski measure, allowing one to express the kinematical Hilbert space as an $L^{2}$ space. The later work [14] by Zapata also proved independently lemma 4 of the present paper.

The paper is organized as follows. First we define the kinematics of piecewise linear LQG, motivate a choice of generalized diffeomorphism group and solve the diffeomorphism constraint. The unitary map between the diffeomorphism-invariant Hilbert spaces in pILQG and LQG is then explicitly constructed and proven in section 3. Equivalence of diffeomorphism-invariant operators in the two frameworks, and the equivalence of dynamics in the two frameworks, is proven in section 4. Everything proven up to this point applies for spatial manifolds with arbitrary (three-dimensional) topology. The exact embeddings of LQC into plLQG of the type motivated in [4, 5] are then explicitly reviewed in section 5 , and at the end of this section, the resulting formal expressions for the embeddings of LQC into LQG at the diffeomorphism-invariant level are given. We then close with a brief discussion. 


\section{Piecewise linear loop quantum gravity}

\subsection{Kinematics}

Much of this section overlaps with the work of Zapata [13].

For simplicity (and because it is the case relevant for the application to cosmology), we assume that space, $M$, is topologically $\mathbb{R}^{3}$, and we equip $M$ with a fixed, flat frame bundle connection $\partial_{a}$. This flat connection gives us a notion of 'straightness' and fixes a specific piecewise linear structure on $M$. More general topologies are also possible, as well as different piecewise linear structures-we refer the reader to appendix A for the definitions in the general case. All of the arguments and results in this paper hold also in the general case (for $M$ three dimensional) without change, except of course in the section on cosmology, where homogeneity and isotropy dictate the topology and piecewise linear structure of $M$.

Let $\mathcal{A}$ denote the space of smooth $S U$ (2) connections on $M$. The classical phase space is parametrized by such a connection $A_{a}^{i}$ and a densitized triad field $\tilde{E}_{i}^{a}$. (Here $A_{a}^{i}$ denotes the components of the $S U$ (2) connection with respect to the basis $\tau_{i}:=-\frac{i}{2} \sigma_{i}$ of the Lie algebra $\mathfrak{s u}(2)$.) The Poisson brackets are given by

$$
\left\{A_{a}^{i}(x), \tilde{E}_{j}^{b}(y)\right\}=8 \pi \gamma G \delta_{j}^{i} \delta_{a}^{b} \delta^{3}(x, y)
$$

where $G$ is Newton's constant, and $\gamma \in \mathbb{R}^{+}$is the Barbero-Immirzi parameter.

Next one specifies the basic variables. The algebra of elementary configuration variables is chosen to consist of (real analytic ${ }^{5}$ ) functions of finite numbers of holonomies of the connection $A_{a}^{i}$ along piecewise straight edges; we will also use the term piecewise linear for such edges. We call these functions piecewise linear cylindrical and the space of such functions is denoted by Cyl. The elementary momentum variables are taken to be the fluxes on piecewise flat surfaces ${ }^{6}$. Given a surface $S$ and a function $f: S \rightarrow \mathfrak{s u}(2)$, the corresponding flux is

$$
E(S, f):=\int_{S} f^{i} \tilde{E}_{i}^{a} n_{a} \mathrm{~d} \sigma_{1} \mathrm{~d} \sigma_{2}
$$

where $n_{a}:=\epsilon_{a b c} \frac{\partial x^{b}}{\partial \sigma_{1}} \frac{\partial x^{c}}{\partial \sigma_{2}},\left(\sigma_{1}, \sigma_{2}\right)$ are arbitrary coordinates on $S, x^{a}$ are arbitrary coordinates on the spatial manifold and $\epsilon_{a b c}$ denotes the fully anti-symmetric symbol (i.e. the Levi-Civita tensor of density weight -1 ).

Next let us introduce some structures to give a more useful characterization of Cyl. We first define a piecewise linear path to be a continuous path $e:[0,1] \rightarrow M$ consisting of a finite number of segments, each segment being geodesic with respect to $\partial_{a}$ (but not necessarily affinely parametrized.) We then define a piecewise linear edge to be an equivalence class of piecewise linear paths, where two piecewise linear paths are equivalent if they are related by a reparametrization, or addition or removal of a 'trivial' segment of the form $\left(\delta \circ \delta^{-1}\right){ }^{7} \mathrm{We}$ next define a piecewise linear graph to be a finite, ordered set of piecewise linear edges. Let $\underline{\Gamma}$ denote the space of piecewise linear graphs. With these definitions, any element $\Phi$ of $\underline{\mathrm{Cyl}}$ can be written in the form

$$
\Phi[A]=F\left(A\left(e_{1}\right), \ldots, A\left(e_{n}\right)\right)
$$

for some piecewise linear graph $\left(e_{1}, \ldots, e_{n}\right) \in \underline{\Gamma}$, and some real-analytic function $F$ :

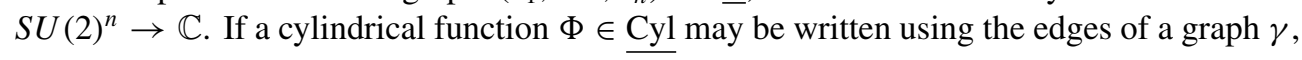

\footnotetext{
5 As always, one has some freedom in the precise definition of cylindrical functions. This definition will be convenient for section 5 .

6 We may also include the fluxes on arbitrary piecewise analytic surfaces, but nothing is thereby gained, and using piecewise flat surfaces is more in the spirit of piecewise linear loop quantum gravity as presented here.

7 Thus, two paths are equivalent iff they yield the same holonomies.
} 
we say $\Phi$ is cylindrical with respect to $\gamma$. We denote by $\mathrm{Cyl}_{\gamma}$ the space of functions cylindrical with respect to $\gamma$.

We next define an inner product $\langle\cdot, \cdot\rangle$ on Cyl in the same way as in standard LQG: given $\Psi, \Phi \in \mathrm{Cyl}$, we find a graph $\gamma$ large enough so that $\Psi, \Phi \in \mathrm{Cyl}_{\gamma}$, and then define the inner product between $\Psi$ and $\Phi$ using the Haar measure on $S U$ (2). As in LQG, this inner product is independent of the ambiguity in the choice of $\gamma$. For each $\gamma$ let $\mathcal{H}_{\gamma}$ denote the Cauchy completion of $\mathrm{Cyl}_{\gamma}$, and let $\underline{\mathcal{H}}$ denote the Cauchy completion of $\mathrm{Cyl}$, in this inner product.

We then construct a representation of the basic algebra on $(\overline{\mathrm{Cyl},}\langle\cdot, \cdot\rangle)$. The configuration algebra Cyl is represented by multiplication. The operators corresponding to the momentum degrees of freedom are then defined via the classical Poisson bracket

$$
\widehat{E(S, f)} \Phi=\mathrm{i}\{E(S, f), \Phi\}
$$

which ensures that the commutators of elements of Cyl and the fluxes match the corresponding Poisson brackets. The multiplicative Cyl operators are bounded because each element of Cyl, as a continuous function of a finite number of $S U(2)$ holonomies, is bounded due to the compactness of $S U(2)$. These multiplicative operators thus extend to all of $\underline{\mathcal{H}}$ by the BLT theorem. The flux operators, equipped with domain Cyl, form essentially self-adjoint operators, which therefore extend uniquely to self-adjoint operators on $\underline{\mathcal{H}}$. The resulting representation of the basic observables then reflects correctly not only the poisson brackets but also the correct adjointness relations. This is the elementary quantization.

After the quantization of the elementary operators, other geometrical operators corresponding to length, area and volume can be constructed in the same way as in standard LQG $[1,2,10,17]$, all with the same spectra. The Gauss constraint is defined in the same way as in standard LQG $[1,2]$ and is just as easy to solve, yielding as a solution space $\underline{\mathcal{H}}_{G} \subset \underline{\mathcal{H}}$, consisting in the Cauchy completion of the span of gauge-invariant spin-network states [1, 2], this time with the graphs restricted to be in $\underline{\Gamma}$.

\subsection{Solution to the diffeomorphism contraint}

Next, let us discuss the solution to the diffeomorphism constraint. Central to this is the selection of a generalization of the group of diffeomorphisms to be used in quantum theory. Once this generalization is selected, we will simply use the group averaging strategy of $[1,18]$ to solve the constraint.

2.2.1. The choice of diffeomorphism gauge group. Let Diff denote the group of generalized diffeomorphisms to be used. We first stipulate several requirements of Diff, which will lead us to a choice for the group. First, we stipulate that the generalized diffeomorphisms at least consist of bijective maps of space onto itself ${ }^{8}$. Second, each element of Diff must map all piecewise linear edges to piecewise linear edges, so that it has a well-defined action on $\underline{\Gamma}$, the set of piecewise linear graphs. These requirements, however, are so far not enough: if we were to only require these, one could map any graph into any other with such a 'generalized diffeomorphism', and, if one follows the prescription of [1, 18], one would be led to a solution space with only a single state. Therefore, we furthermore stipulate that the maps be homeomorphisms. A natural choice satisfying the above requirements is the group of piecewise linear homeomorphisms. To define the notion of a piecewise linear homeomorphism, we must first review the definition of a simplicial complex [19]. First, we note that the fixed connection

8 If one were to solve the Gauss and diffeomorphism constraints together, this would be equivalent to requiring that the generalized principal bundle automorphisms to be used should at least consist of maps from the principal bundle to itself that preserve all structure of the principal bundle except possibly topology and differentiable structure. 
$\partial_{a}$ endows $M$ with a natural affine structure. Let us for convenience arbitrarily pick an origin $O \in M$, and use this to make $M$ into a vector space, so that addition and real scalar multiplication are defined in $M$. None of the definitions or constructions below will depend on the choice of $O$.

A set of points $\left\{a_{0}, \ldots, a_{n}\right\} \subset M$ is said to be independent if they do not lie within any common $(n-1)$-dimensional plane in $M$. Given such a set of $n+1$ independent points, we define the $n$-simplex $\sigma$ spanned by $a_{0}, \ldots, a_{n}$ to be the set of all points $x \in M$ such that

$$
x=\sum_{i=0}^{n} t_{i} a_{i}
$$

for some $t_{0}, \ldots, t_{n} \in \mathbb{R}$ all non-negative, satisfying $\sum_{i=0}^{n} t_{i}=1 . n$ is called the dimension of $\sigma$. In common language, a 0 -simplex is a point, a 1 -simplex is a line segment, a 2 -simplex is a triangle and a 3-simplex is a tetrahedron.

Next we define the generalized notion of 'face'. Given an $n$-simplex $\sigma$ spanned by a set of points $\left\{a_{0}, \ldots, a_{n}\right\}$, the simplex spanned by a subset of these points is called a face of $\sigma$. In particular, every simplex is a face of itself; a face of a simplex $\sigma$ that is not equal to $\sigma$ is called a proper face. Thus, in this generalized sense, the proper 'faces' of a tetrahedron consist of all the triangular faces in the usual sense, all the edges and all four vertices. The proper 'faces' of a triangle consist of its three edges, three vertices, etc.

We can now define a simplicial complex $K$ to be a (locally finite) collection of simplices such that

(1) every face of a simplex of $K$ is in $K$,

(2) the intersection of any two simplices of $K$ is a face of each of them.

The maximal simplex dimension occurring in $K$ is called the dimension of $K$.

Finally, a homeomorphism $F$ from an $n$-dimensional manifold $M$ onto an $n$-dimensional manifold $N$ is called piecewise linear if there exist simplicial complexes $K$ and $L$, covering all of $M$ and $N$, respectively, such that $v_{0}, \ldots, v_{m}$ span a simplex of $K$ if and only if $F\left(v_{0}\right), \ldots, F\left(v_{m}\right)$ span a simplex of $L$, and such that for each $\left\{v_{0}, \ldots, v_{n}\right\}$ spanning an $n$-simplex in $K$,

$$
F\left(\sum_{i=0}^{n} t_{i} v_{i}\right)=\sum_{i=0}^{n} t_{i} F\left(v_{i}\right)
$$

for all $t_{i} \geqslant 0$ satisfying $\sum_{i=0}^{n} t_{i}=1$. That is, $F$ maps simplices of $K$ into simplices of $L$ in a continuous way, such that $F$ is linear within each $n$-simplex ${ }^{9}$.

The piecewise linear homeomorphisms are essentially the piecewise linear analog of the stratified analytic diffeomorphisms advocated in [9] and described in [20, 21] (see also $[14,22])$. In the analytic framework, however, one has more choices: one can, for example, require that the generalized diffeomorphisms be at least differentiable. The analog of such a requirement can, however, not be satisfied in the piecewise linear framework: the only differentiable piecewise linear maps are globally linear. But the group of globally linear maps is too small, in the sense that there are no local degrees of freedom therein that would allow one to approximate arbitrary diffeomorphisms. Piecewise linear homeomorphisms, by contrast, do for example in the sense proven in the classic simplicial approximation theorem [23]. Furthermore, if one were to choose globally linear maps as the group of 'generalized diffeomorphisms', then linear relations among the tangent vectors of even distant, disconnected edges would survive as information at the 'diffeomorphism-invariant' level. This would prevent any possible relation with any analytic LQG framework so far proposed.

9 In the language of [19], a piecewise linear homeomorphism is a simplicial homeomorphism from some simplicial complex $K$ to another $L$. 
2.2.2. Construction of the diffeomorphism-invariant Hilbert space. With the foregoing choice of Diff, let us proceed to construct the solution to the diffeomorphism constraint. For this purpose, we introduce some further definitions. First, if two graphs $\gamma_{1}, \gamma_{2} \in \Gamma$ differ only by a permutation of edges or reversal of edge orientations, we call them probe equivalent. The probe equivalence class of a graph $\gamma$ we write as $[\gamma]_{p r}$. Let $\underline{\Gamma}_{\mathrm{pr}}$ denote the space of such probe equivalence classes in $\underline{\Gamma}$. Next, for each $\gamma \in \underline{\Gamma}$, let $\mathcal{H}_{\gamma}^{\prime}$ denote the orthogonal complement, in $\mathcal{H}_{\gamma}$, of the span of all functions that are constant on at least one edge of $\gamma$. Then, as in [1],

$$
\underline{\mathcal{H}}=\oplus_{[\gamma]_{\mathrm{pr}} \in \underline{\Gamma}_{\mathrm{pr}}} \mathcal{H}_{\gamma}^{\prime} .
$$

Furthermore, let $\mathrm{Cyl}_{\gamma}^{\prime}:=\mathcal{H}_{\gamma}^{\prime} \cap \mathrm{Cyl}$. Lastly we define some subgroups of our chosen generalized diffeomorphisms. For each $\gamma \in \underline{\Gamma}$, let $\underline{\mathrm{Diff}}_{\gamma}$ be the set of elements in Diff mapping $\gamma$ back into its probe equivalence class. Let $\underline{\text { TDiff }}_{\gamma}$ be the set of elements in $\underline{\text { Diff }}$ fixing $\gamma$, so that they preserve each edge of $\gamma$ including orientation. Let $\underline{G S}_{\gamma}:=\underline{\text { Diff }}_{\gamma} / \underline{\text { TDiff }}_{\gamma}$ where the division is taken with respect to the left action.

For each $\gamma \in \underline{\Gamma}$, define $\underline{P}_{\text {diff, } \gamma}$ as the group averaging map $[18,1]$ from $\mathcal{H}_{\gamma}^{\prime}$ to the subspace invariant under $\underline{G S}_{\gamma}:{ }^{10}$

$$
\underline{P}_{\mathrm{diff}, \gamma} \Psi_{\gamma}:=\frac{1}{\mid \underline{G S_{\gamma} \mid}} \sum_{\varphi \in \underline{G S}_{\gamma}} \varphi^{*} \Psi_{\gamma} .
$$

For each $\Psi_{\gamma} \in \mathrm{Cyl}_{\gamma}^{\prime}$, define $\underline{\eta}\left(\Psi_{\gamma}\right) \in \underline{\mathrm{Cyl}^{*}}$ by

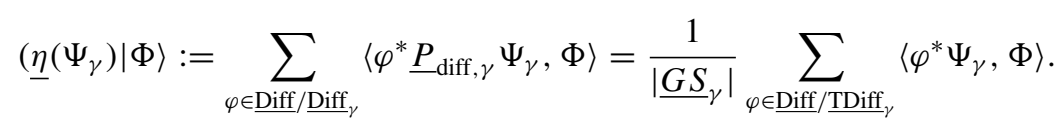

Piecing together these maps for the various $\gamma \in \underline{\Gamma}$ defines a map $\underline{\eta}: \underline{\mathrm{Cyl}} \rightarrow \mathrm{Cyl}^{*}$. This is the rigging map for solving the diffeomorphism constraint for piecewise linear $\overline{\mathrm{LQG}}$. The space of 'test functions' at the diffeomorphism-invariant level is then

$$
\underline{\mathrm{Cyl}}_{\mathrm{diff}}^{*}:=\operatorname{Im} \underline{\eta} \text {. }
$$

The inner product on this space is defined as follows: for $\underline{\eta} \Psi, \underline{\eta} \Phi \in \operatorname{Im} \underline{\eta}$,

$$
\langle\underline{\eta} \Psi, \underline{\eta} \Phi\rangle:=(\underline{\eta} \Psi|\Phi\rangle .
$$

The Cauchy completion of $\underline{\mathrm{Cyl}}_{\text {diff }}^{*}$ with respect to the above inner product we denote by $\underline{\mathcal{H}}_{\text {diff }}$.

The solution to both the Gauss and diffeomorphism constraints is constructed by first defining $\underline{\mathrm{Cyl}}_{\mathrm{diff}, G}^{*}:=\underline{\eta}\left[\underline{\mathrm{Cyl}} \cap \underline{\mathcal{H}}_{G}\right] \subset \underline{\mathrm{Cyl}}_{\mathrm{diff}}^{*}$, and then Cauchy completing to obtain $\underline{\mathcal{H}}_{\text {diff }, G} \subset \underline{\mathcal{H}}_{\text {diff }}$.

\section{Equivalence of piecewise linear LQG with analytic LQG at the diffeomorphism-invariant level}

In this section we prove that the diffeomorphism-invariant Hilbert space for piecewise linear LQG is naturally isomorphic to the diffeomorphism-invariant Hilbert space of standard LQGprovided that for standard LQG one uses a generalized diffeomorphism group such as that advocated in [9].

We begin by proving the key lemma about piecewise linear LQG allowing the equivalence. Define a 'graph knot-class' as a homeomorphism-equivalence class of topologically embedded graphs. The key lemma essentially states that Diff equivalence classes of piecewise linear graphs are graph knot-classes. Because the analog of this is also true for piecewise analytic

${ }^{10}$ In lemma 5, we will show that $\underline{P}_{\text {diff, } \gamma}$ is equal to $P_{\text {diff, } \gamma}$ in [1]. 
LQG with the choice of diffeomorphism group advocated in [9], one already has a hint of the equivalence of the two theories at the diffeomorphism-invariant level. However, to rigorously prove the equivalence, more must be done, and the subsequent part of this section is devoted to this task.

First we give several definitions. Given a simplicial complex $K$, a subcomplex $K^{\prime}$ is any subset of $K$ such that $K^{\prime}$ is again a simplicial complex. (Note that it is possible for the dimension of $K^{\prime}$ to be less than that of $K$ ). Second, a complex $\tilde{K}$ is said to be a subdivision of a complex $K$ if every simplex of $\tilde{K}$ is contained in a simplex of $K$, and every simplex of $K$ is a union of simplices in $\tilde{K}$. Third, given a simplicial complex $K$, we denote the union of simplices in $K$ by

$$
|K|:=\cup_{A \in K} A,
$$

called the polyhedron underlying $K$. Lastly, we define a piecewise linear graph $\gamma$ and a 1complex $X$ to be compatible if the image of $\gamma$ (which we denote by $|\gamma|$ ) equals $|X|$. By breaking up each edge of a piecewise linear $\gamma$ into its straight pieces, and taking the set of these line segments and all their endpoints, one obtains the simplest 1-complex compatible with $\gamma$. By subdividing the edges further, one obtains other compatible 1-complexes.

We begin by stating a lemma, which is almost identical to (4.4) of [24].

Lemma 1 (Almost (4.4) of Brown [24]). Let $K$ and $L$ be 3-complexes and let $K_{1}$ and $L_{1}$ be one-dimensional subcomplexes of $K$ and $L$ respectively. Suppose $f:|K| \rightarrow|L|$ is a homeomorphism such that $f\left(\left|K_{1}\right|\right)=\left|L_{1}\right|$. Then there exists an isotopy $g_{t}:|K| \rightarrow|L|$ such that

(i) $g_{0}=f$;

(ii) there exist subdivisions $\tilde{K}, \tilde{L}, \tilde{K}_{1}, \tilde{L}_{1}$ of $K, L, K_{1}, L_{1}$ respectively such that
(a) $\tilde{K}_{1}$ and $\tilde{L}_{1}$ are subcomplexes of $\tilde{K}$ and $\tilde{L}$, respectively,
(b) $g_{t}$ maps $\tilde{K}_{1}$ onto $\tilde{L}_{1}$ for all $t$, and
(c) $g_{1}$ is piecewise linear on $\tilde{K}_{1}$.

Proof. The proof is exactly the same as that given for (4.4) in [24]; only the statement of the lemma differs.

We use the above in proving the following key lemma. A generalized version of the Hauptvermutung of algebraic topology for 3-complexes, proved in 1969 [24], plays a key role in the following proof.

Lemma 2. If $\gamma, \gamma^{\prime} \in \underline{\Gamma}$ admit a homeomorphism $\xi: M \rightarrow M$ such that $\gamma^{\prime}=\xi \cdot \gamma$, then

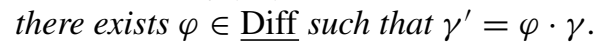

Proof. First, by lemma 12 in the appendix, there exist simplicial complexes $K$ and $L$, each triangulating all of $M$, such that $K$ contains a one-dimensional subcomplex $K_{1}$ compatible with $\gamma$, and $L$ contains a one-dimensional subcomplex $L_{1}$ compatible with $\gamma^{\prime}$. Because $\xi$ maps $\gamma$ to $\gamma^{\prime}$, it maps $\left|K_{1}\right|$ to $\left|L_{1}\right|$. We now invoke lemma 1 above; it provides us with subdivisions $\tilde{K}, \tilde{L}, \tilde{K}_{1}, \tilde{L}_{1}$ of $K, L, K_{1}, L_{1}$ such that $\tilde{K}_{1}$ and $\tilde{L}_{1}$ are subcomplexes of $\tilde{K}$ and $\tilde{L}$, and an isotopy $\xi_{t}: M \rightarrow M$ such that (i) $\xi_{0}=\xi$, (ii) $\xi_{t}$ maps $\tilde{K}_{1}$ to $\tilde{L}_{1}$ for all $t$ and (iii) $\xi_{1}$ is piecewise linear on $\tilde{K}_{1}$.

The 3 -complexes $\tilde{K}$ and $\tilde{L}$, the subcomplex $\tilde{K}_{1}$ of $\tilde{K}$ and the homeomorphism $\xi_{1}$ now satisfy the hypotheses of theorem (4.8) of [24], which implies the existence of an isotopy $\varphi_{t}: M \rightarrow M$ such that (i) $\varphi_{0}=\xi_{1}$, (ii) $\varphi_{1}$ is piecewise linear and (iii) $\left.\varphi_{t}\right|_{\left|\tilde{K}_{1}\right|}=\left.\xi_{1}\right|_{\left|\tilde{K}_{1}\right|}$ for all $t$. 
Now, as already noted, $\xi_{t}$ maps $\tilde{K}_{1}$ as a 1-complex onto $\tilde{L}_{1}$ as a 1-complex for all $t$. That is, $\xi_{t}$ maps each simplex of $\tilde{K}_{1}$ to a corresponding simplex of $\tilde{L}_{1}$ in an onto fashion; this mapping is furthermore 1-1 from the injectivity of $\xi_{t}$. Now, because $\tilde{K}_{1}$ is a subdivision of $K_{1}$, and $K_{1}$ is compatible with $\gamma, \tilde{K}_{1}$ is also compatible with $\gamma$, so that each edge of $\gamma$ is a union of simplices in $\tilde{K}_{1}$. Likewise, each edge of $\gamma^{\prime}$ is a union of simplices in $\tilde{L}_{1}$. It follows that, for all $t, \xi_{t}$ maps each edge of $\gamma$ onto a corresponding edge of $\gamma^{\prime}$ in a 1-1 and onto fashion. The continuity of $\xi_{t}$ in $t$ ensures that $\xi_{t}$ always maps each edge of $\gamma$ to the same edge of $\gamma^{\prime}$ for all $t$. Furthermore, recall that $\xi_{0}=\xi$ maps the orientation of each edge in $\gamma$ correctly into the orientation of the corresponding edge in $\gamma^{\prime}$; the continuity of $\xi_{t}$ in $t$ ensures that $\xi_{t}$ does the same for all $t$. Thus, for all $t, \xi_{t}$ maps $\gamma$ onto $\gamma^{\prime}$ as a graph. This is in particular true for $\xi_{1}$; property (iii) of $\varphi_{t}$ then implies that this is also true for $\varphi_{t}$ for all $t . \varphi:=\varphi_{1}$ thus provides a piecewise linear homeomorphism, i.e. an element of Diff, mapping $\gamma$ to $\gamma^{\prime}$, as desired.

The above lemma in particular implies that $\underline{\Gamma / \text { Diff }}$ is isomorphic to the set of graph knot-classes. This follows from the fact that the converse of the above lemma is trivially true and that every graph knot-class has a representative in $\underline{\Gamma}$ (as is not hard to see).

Next, let $\Gamma$ denote the set of piecewise analytic graphs: that is, graphs with a finite number of oriented compact edges, each of which can be subdivided into a finite number of analytic curves.

Definition (Probe equivalent). When two graphs $\gamma, \gamma^{\prime} \in \Gamma$ differ only by a permutation of edges or reversal of edge orientations, we say that $\gamma$ and $\gamma^{\prime}$ are probe equivalent. We write the probe equivalence class of a graph $\gamma$ as $[\gamma]_{\mathrm{pr}}$.

Let $\Gamma_{\mathrm{pr}}$ denote the set of probe equivalence classes in $\Gamma$, as we have let $\Gamma_{\mathrm{pr}}$ denote the set of probe equivalence classes in $\underline{\Gamma}$.

Let Diff denote the class of diffeomorphisms which one wishes to use to solve the diffeomorphism constraint in the piecewise analytic framework. There are multiple proposals for such a class of diffeomorphisms in the literature. We make two assumptions about the choice of Diff.

Assumption 1. Diff is a subgroup of the homeomorphisms of $M$.

Assumption 2. If $\gamma, \gamma^{\prime} \in \Gamma$ are such that $\gamma^{\prime}=\xi \cdot \gamma$ for some homeomorphism $\xi: M \rightarrow M$, then there exists $\varphi \in$ Diff such that $\gamma^{\prime}=\varphi \cdot \gamma$.

These assumptions imply that $\Gamma /$ Diff too is isomorphic to the set of graph knot-classes (using reasoning similar to that in the case of $\underline{\Gamma} / \underline{\text { Diff }})$. Note that if Diff is chosen to be the stratified analytic diffeomorphisms [20, 21] as advocated in [9], then both assumptions (1) and (2) are satisfied - assumption (1) is immediate, and assumption (2) follows from lemma 4 in [9]. ${ }^{11}$

Note, however, that this choice, like any choice of Diff satisfying the above assumptions, necessarily includes elements that are non-differentiable. This is due to the existence of certain 'continuous moduli' [22] describing the differentiable structure of graphs at vertices. Assumption 2 implies that Diff, like the homeomorphisms, must act transitively on the space of these continuous moduli for fixed valence; bi-differentiable maps, by contrast, preserve such moduli. A consequence of this is that the Ashtekar-Lewandowski ordering of the volume operator $[1,2,11]$, as it involves these continuous moduli, is necessarily non-covariant with respect to any choice of Diff satisfying the above assumptions. This in turn means that, if one is to define Hamiltonian and master constraints via the usual construction $[15,16]$, one needs

${ }^{11}$ Using the analytic differentiability class. 
to use the Rovelli-Smolin volume operator $[1,2,10]$ instead of the Ashtekar-Lewandowski one; in section 4 we shall do this ${ }^{12}$.

Finally, let $\mathcal{A}$ denote the space of smooth $S U(2)$ connections on $M$. In defining analytic LQG and its diffeomorphism-invariant Hilbert space, we follow the presentation in [1]. The structures necessary to construct the diffeomorphism-invariant Hilbert space are the following:

Definition (Piecewise analytic LQG structures).

(1) Given a graph $\gamma \in \Gamma$, let $\mathrm{Cyl}_{\gamma}$ denote the set of functions on $\mathcal{A}$ cylindrical with respect to $\gamma$ (note that for $\gamma \in \underline{\Gamma}$, this is consistent with the prior definition of $\mathrm{Cyl}_{\gamma}$ ). Let $\mathrm{Cyl}:=\cup_{\gamma} \mathrm{Cyl}_{\gamma}$.

(2) Let $\langle$,$\rangle denote the standard inner product on \mathrm{Cyl}$ defined using the Haar measure on SU (2) $[1,2]$. Let $\mathcal{H}_{\gamma}$ and $\mathcal{H}$ denote the Cauchy completions of $\mathrm{Cyl}_{\gamma}$ and $\mathrm{Cyl}$, respectively, with respect to $\langle$,$\rangle .$

(3) Let $\mathcal{H}_{G}$ denote the solution space to the Gauss constraint, consisting as usual in the Cauchy completion of the span of gauge-invariant spin networks [1, 2].

(4) For each $\gamma \in \Gamma$, let $\mathcal{H}_{\gamma}^{\prime}$ denote the orthogonal complement, in $\mathcal{H}_{\gamma}$, of the span of all functions that are constant on at least one edge of $\gamma$, so that, as in [1], $\mathcal{H}=\oplus_{[\gamma] \in \Gamma_{\mathrm{pr}}} \mathcal{H}_{\gamma}^{\prime}$. Let $\mathrm{Cyl}_{\gamma}^{\prime}:=\mathrm{Cyl} \cap \mathcal{H}_{\gamma}^{\prime}$. (For $\gamma \in \bar{\Gamma}$, these definitions are again consistent with the ones in the piecewise linear framework.)

(5) For each $\gamma \in \Gamma$, let Diff ${ }_{\gamma}$ be the set of elements in Diff mapping $\gamma$ back into its probe equivalence class. Let $\mathrm{TDiff}_{\gamma}$ be the set of elements in Diff that fix $\gamma$-i.e. that preserve each edge of $\gamma$, including orientation. So defined, Diff $\gamma$ is precisely the subset of Diff preserving $\mathrm{Cyl}_{\gamma}^{\prime}$ under pullback, and $\mathrm{TDiff}_{\gamma}$ is precisely the subset of Diff that acts as the identity on $\mathrm{Cyl}_{\gamma}^{\prime}$ under pullback. Let $G S_{\gamma}:=\operatorname{Diff}_{\gamma} / \mathrm{TDiff}_{\gamma}$ where the division is taken with respect to the left action.

(6) For each $\gamma \in \Gamma$, define $P_{\mathrm{diff}, \gamma}$ as the group averaging map from $\mathcal{H}_{\gamma}^{\prime}$ to the subspace invariant under $G S_{\gamma}$ :

$$
P_{\mathrm{diff}, \gamma} \Psi_{\gamma}:=\frac{1}{\left|G S_{\gamma}\right|} \sum_{\varphi \in G S_{\gamma}} \varphi^{*} \Psi_{\gamma}
$$

For each $\Psi_{\gamma} \in \mathrm{Cyl}_{\gamma}^{\prime}$, define $\eta\left(\Psi_{\gamma}\right) \in \mathrm{Cyl}^{*}$ by

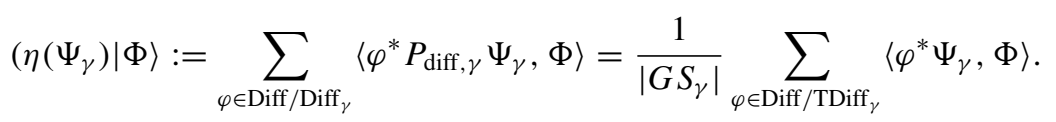

Piecing these together for all $\gamma$ defines a map $\eta: \mathrm{Cyl} \rightarrow \mathrm{Cyl}^{*}$. This is the rigging map for the theory, as defined in [1] (see also [18], and the related [22]).

(7) $\mathrm{Cyl}_{\mathrm{diff}}^{*}:=\operatorname{Im} \eta$. For $\eta \Psi, \eta \Phi \in \operatorname{Im} \eta$,

$$
\langle\eta \Psi, \eta \Phi\rangle:=(\eta \Psi|\Phi\rangle .
$$

$\mathcal{H}_{\text {diff }}$ is then defined to be the Cauchy completion of $\mathrm{Cyl}_{\mathrm{diff}}^{*}$ with respect to this inner product. The completion $\mathcal{H}_{\mathrm{diff}, G}$ of the subspace $\mathrm{Cyl}_{\mathrm{diff}, G}^{*}:=\eta\left[\mathrm{Cyl} \cap \mathcal{H}_{G}\right] \subset \mathcal{H}_{\mathrm{diff}}$ is then the solution to both the Gauss and diffeomorphism constraints.

We next prove a few important lemmas which we use.

\footnotetext{
${ }^{12}$ We do not wish to imply that the Rovelli-Smolin ordering is 'more correct'. Rather, this paper simply shows an interesting result that can be obtained if one uses the Rovelli-Smolin ordering.
} 


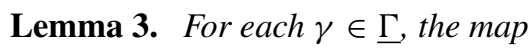

$$
\begin{aligned}
& F: \underline{G S_{\gamma}} \rightarrow G S_{\gamma} \\
& \varphi \circ\left[\underline{\mathrm{TDiff}}_{\gamma}\right] \mapsto \varphi \circ\left[\mathrm{TDiff}_{\gamma}\right]
\end{aligned}
$$

is well defined, and is an isomorphism, showing $\underline{G S}_{\gamma} \cong G S_{\gamma}$.

\section{Proof.}

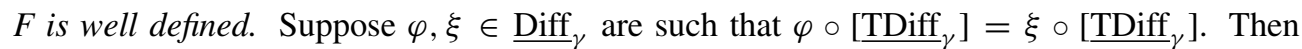
$\varphi^{-1} \circ \xi \in \underline{\operatorname{TDiff}}_{\gamma}$, whence $\varphi^{-1} \circ \xi \in \operatorname{TDiff}_{\gamma}$, so that $\varphi \circ\left[\operatorname{TDiff}_{\gamma}\right]=\xi \circ\left[\operatorname{TDiff}_{\gamma}\right]$, proving $F$ well defined.

$F$ is a homomorphism. This is immediate from the definition of multiplication in the two quotient groups.

$F$ is injective. Suppose $\varphi, \xi \in \underline{\operatorname{Diff}}_{\gamma}$ are such that $\varphi \circ\left[\operatorname{TDiff}_{\gamma}\right]=\xi \circ\left[\operatorname{TDiff}_{\gamma}\right]$. Then

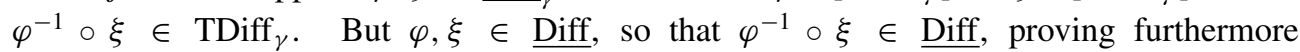
$\varphi^{-1} \circ \xi \in \underline{\mathrm{TDiff}}_{\gamma}$. It follows $\varphi \circ\left[\underline{\mathrm{TDiff}}_{\gamma}\right]=\xi \circ\left[\underline{\mathrm{TDiff}}_{\gamma}\right]$, proving injectivity.

$F$ is surjective. Let $\varphi \circ\left[\operatorname{TDiff}_{\gamma}\right] \in G S_{\gamma}$ be given. Let $\gamma^{\prime}:=\varphi \cdot \gamma$. As $\varphi \in \operatorname{Diff}_{\gamma}, \gamma^{\prime}$ is probe equivalent to $\gamma$ and so is also in $\underline{\Gamma}$. Furthermore, $\varphi$ is in particular a homeomorphism, allowing

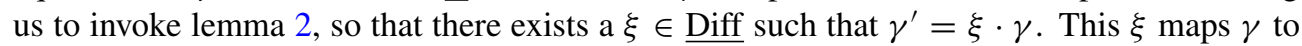
$\gamma^{\prime}$, a graph probe equivalent to $\gamma$, whence $\xi \in \underline{\mathrm{Diff}}_{\gamma}$. Furthermore, $\left(\varphi^{-1} \circ \xi\right) \gamma=\gamma$, so that $\varphi^{-1} \circ \xi \in \operatorname{TDiff}_{\gamma}$, whence $\xi \circ\left[\operatorname{TDiff}_{\gamma}\right]=\varphi \circ$ [TDiff $_{\gamma}$ ]. Thus, $F\left(\xi \circ\left[\right.\right.$ TDiff $\left.\left._{\gamma}\right]\right)=\varphi \circ$ [TDiff $\left._{\gamma}\right]$, proving surjectivity.

\section{Lemma 4.}

(1) Given $\gamma \in \Gamma$, there exists $\varphi \in$ Diff such that $\varphi \cdot \gamma \in \underline{\Gamma}$.

(2) Given $\Psi \in \mathrm{Cyl}$, there exists $\tilde{\varphi} \in$ Diff such that $\tilde{\varphi}^{*} \Psi \in \mathrm{Cyl}$.

\section{Proof.}

Proof of (1). Let $\alpha$ be any element of $\underline{\Gamma}$ with the same knot-class as $\gamma$ (it is easy to see that one can construct an element of $\underline{\Gamma}$ with any desired knot-class), and choose the ordering and orientation of the edges of $\alpha$ such that $\alpha=\xi \cdot \gamma$ for some homeomorphism $\xi: M \rightarrow M$. Assumption 2 implies that there exists $\varphi \in$ Diff such that $\alpha=\varphi \cdot \gamma$.

Proof of (2). As $\Psi \in \mathrm{Cyl}, \Psi \in \mathrm{Cyl}_{\gamma}$ for some $\gamma \in \Gamma$. From part (1), there exists $\varphi \in \operatorname{Diff}$ such that $\varphi \cdot \gamma \in \underline{\Gamma}$, so that $\left(\varphi^{-1}\right)^{*} \Psi \in \underline{\mathrm{Cyl}}$.

Because $\underline{\mathrm{Cyl}} \subset \mathrm{Cyl}$, we have a natural map $\mathcal{I}: \mathrm{Cyl}^{*} \rightarrow \underline{\mathrm{Cyl}^{*}}$ defined by

$$
(\mathcal{I} \Psi|\Phi\rangle:=(\Psi|\Phi\rangle
$$

for all $\Psi \in \mathrm{Cyl}^{*}$ and $\Phi \in \underline{\mathrm{Cyl}}$.

\section{Lemma 5.}

(1) For $\gamma \in \underline{\Gamma,} P_{\text {diff, } \gamma}=\underline{P}_{\text {diff, } \gamma}$.

(2) For $\Psi \in$ Cyl, $\mathcal{I} \eta \Psi=\underline{\eta} \Psi$. 


\section{Proof.}

Proof of (1). We use the isomorphism $F$ from lemma 3. It is immediate from its definition that, for $\Psi \in \mathrm{Cyl}_{\gamma}^{\prime}$ and $\xi \in \underline{\operatorname{Diff}}_{\gamma} / \underline{\mathrm{TDiff}}_{\gamma}, F(\xi)^{*} \Psi=\xi^{*} \Psi$. Using $F$ and this fact,

$$
P_{\mathrm{diff}, \gamma} \Psi:=\frac{1}{\left|G S_{\gamma}\right|} \sum_{\varphi \in G S_{\gamma}} \varphi^{*} \Psi=\frac{1}{\left|\underline{G S_{\gamma}}\right|} \sum_{\varphi \in \underline{G S}_{\gamma}}(F \varphi)^{*} \Psi=\frac{1}{\left|\underline{G S_{\gamma}}\right|} \sum_{\varphi \in \underline{G S}_{\gamma}} \varphi^{*} \Psi=\underline{P}_{\mathrm{diff}, \gamma} \Psi \text {. }
$$

Proof of (2). Using the linearity of $\mathcal{I}, \eta$ and $\eta^{\prime}$, without loss of generality, assume $\Psi \in \mathrm{Cyl}_{\gamma}^{\prime}$ for some $\gamma \in \underline{\Gamma}$. Suppose $\gamma^{\prime} \in \underline{\Gamma}$ and $\Theta \in \mathrm{Cyl}_{\gamma^{\prime}}^{\prime}$ are given.

Case 1: there exists no $\varphi_{o} \in$ Diff such that $\varphi_{o} \cdot \gamma^{\prime}=\gamma$.

Then from (14), $(\mathcal{I} \eta \Psi|\Theta\rangle=0$. But from (9), $(\eta \Psi|\Theta\rangle=0$ as well, so that $(\mathcal{I} \eta \Psi|\Theta\rangle=(\underline{\eta} \Psi|\Theta\rangle=0$.

Case 2: there exists $\varphi_{o} \in$ Diff such that $\varphi_{o} \cdot \gamma^{\prime}=\gamma$.

Then, from lemma 2, there exists $\underline{\varphi}_{O} \in \underline{\text { Diff }}$ such that $\underline{\varphi}_{O} \cdot \gamma^{\prime}=\gamma$. Using the orthogonality of the spaces $\mathcal{H}_{\gamma}^{\prime}$, the middle expression in (14) reduces to

$$
\left(\mathcal{I} \eta \Psi|\Theta\rangle=\left(\eta \Psi|\Theta\rangle=\left\langle\underline{\varphi}_{o}^{*} P_{\text {diff }, \gamma} \Psi, \Theta\right\rangle .\right.\right.
$$

Using part (1) of this lemma, and the same orthogonality of the spaces $\mathcal{H}_{\gamma}^{\prime}$ to simplify the expression for $(\eta \underline{\Psi}|\Theta\rangle$, we also have

$$
\left(\mathcal{I} \eta \Psi|\Theta\rangle=\left\langle\underline{\varphi}_{o}^{*} \underline{P}_{\text {diff, } \gamma} \Psi, \Theta\right\rangle=(\eta \underline{\Psi}|\Theta\rangle .\right.
$$

Thus, $\left(\mathcal{I} \eta \Psi|\Theta\rangle=\left(\underline{\eta} \Psi|\Theta\rangle\right.\right.$ for all $\Theta \in \mathrm{Cyl}_{\gamma^{\prime}}^{\prime}, \gamma^{\prime} \in \underline{\Gamma}$, so that $\mathcal{I} \eta \Psi=\underline{\eta} \Psi$.

Theorem 6. I maps $\mathrm{Cyl}_{\mathrm{diff}}^{*}$ onto $\underline{\mathrm{Cyl}}_{\mathrm{diff}}^{*}$. Furthermore, $\left.\mathcal{I}\right|_{\mathrm{Cyl}}{ }_{\mathrm{diff}}^{*}: \mathrm{Cyl}_{\mathrm{diff}}^{*} \rightarrow \underline{\mathrm{Cyl}}_{\mathrm{diff}}^{*}$ is a unitary isomorphism.

Proof. Proof that $\mathcal{I}\left[\mathrm{Cyl}_{\mathrm{diff}}^{*}\right]=\underline{\mathrm{Cyl}}_{\mathrm{diff}}^{*}$ :

$\subseteq$ : Let $\eta \Psi \in \mathrm{Cyl}_{\text {diff }}^{*}$ be given, so that $\Psi \in \mathrm{Cyl}$. By lemma $4, \exists \xi \in$ Diff s.t. $\xi^{*} \Psi \in$ Cyl. Using the Diff invariance of $\eta$ and part (2) of lemma $5, \mathcal{I} \eta \Psi=\mathcal{I} \eta\left(\xi^{*} \Psi\right)=\eta\left(\xi^{*} \Psi\right)$, which is in $\mathrm{Cyl}_{\text {diff }}^{*}$.

$\supseteq$ : Let $\underline{\eta} \Psi \in \underline{\mathrm{Cyl}}_{\mathrm{diff}}^{*}$ be given, so that $\Psi \in \underline{\mathrm{Cyl}}$. Then $\eta \Psi \in \mathrm{Cyl}_{\mathrm{diff}}^{*}$, and by lemma 5, $\mathcal{I} \eta \Psi=\bar{\eta} \Psi$, so that $\underline{\eta} \Psi \in \mathcal{I}\left[\mathrm{Cyl}_{\mathrm{diff}}^{*}\right]$.

Proof that $\left.\mathcal{I}\right|_{\text {Cyl }_{\mathrm{diff}}^{*}}$ is injective:

Suppose $\eta \Psi, \eta \Phi \in \mathrm{Cyl}_{\text {diff }}^{*}$ are such that $\mathcal{I} \eta \Psi=\mathcal{I} \eta \Phi$. Let $\Theta \in \mathrm{Cyl}$ be given. By lemma 4,

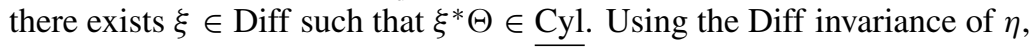

$$
\left(\eta \Psi|\Theta\rangle=\left(\eta \Psi\left|\xi^{*} \Theta\right\rangle=\left(\mathcal{I} \eta \Psi\left|\xi^{*} \Theta\right\rangle=\left(\mathcal{I} \eta \Phi\left|\xi^{*} \Theta\right\rangle=\left(\eta \Phi\left|\xi^{*} \Theta\right\rangle=(\eta \Phi|\Theta\rangle\right.\right.\right.\right.\right.
$$

for all $\Theta \in \mathrm{Cyl}$, whence $\eta \Psi=\eta \Phi$.

Proof that $\left.\mathcal{I}\right|_{\mathrm{Cyl}_{\mathrm{diff}}^{*}}$ is isometric and hence unitary:

Let $\eta \Psi, \eta \Phi \in \mathrm{Cyl}_{\text {diff }}^{*}$ be given, so that $\Psi, \Phi \in \mathrm{Cyl}$. Using lemma 4, there exists $\varphi$ and $\xi$ in Diff such that $\varphi^{*} \Psi, \xi^{*} \Phi \in$ Cyl. Using the Diff invariance of $\eta$ and part (2) of lemma 5, we have

$$
\begin{aligned}
\langle\mathcal{I} \eta \Psi, \mathcal{I} \eta \Phi\rangle & =\left\langle\mathcal{I} \eta\left(\varphi^{*} \Psi\right), \mathcal{I} \eta\left(\xi^{*} \Phi\right)\right\rangle=\left\langle\underline{\eta}\left(\varphi^{*} \Psi\right), \underline{\eta}\left(\xi^{*} \Phi\right)\right\rangle \\
& :=\left(\eta\left(\varphi^{*} \Psi\right)\left|\xi^{*} \Phi\right\rangle=\left(\mathcal{I} \eta\left(\varphi^{*} \Psi\right)\left|\xi^{*} \Phi\right\rangle=\left(\eta\left(\varphi^{*} \Psi\right)\left|\xi^{*} \Phi\right\rangle\right.\right.\right. \\
& =(\eta \Psi|\Phi\rangle=\langle\eta \Psi, \eta \Phi\rangle .
\end{aligned}
$$

The above theorem implies the following. 
Corollary 7. $\mathcal{H}_{\text {diff }}$ and $\underline{\mathcal{H}}_{\text {diff }}$ are isomorphic as Hilbert spaces.

It is then easy to extend the equivalence to the solution spaces solving both the diffeomorphism and Gauss constraints.

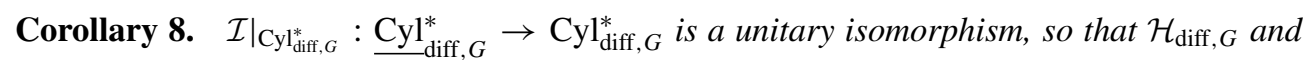
$\underline{\mathcal{H}}_{\mathrm{diff}, G}$ are isomorphic as Hilbert spaces.

Proof. From the injectivity of $\left.\mathcal{I}\right|_{\mathrm{Cy}_{\mathrm{diff}}^{*}}$, we know that $\left.\mathcal{I}\right|_{\mathrm{Cyl}_{\mathrm{diff}, G}^{*}}$ is injective. It thus remains only to prove that $\mathcal{I}$ maps $\mathrm{Cyl}_{\mathrm{diff}, G}^{*}$ onto $\underline{\mathrm{Cy}} \underline{\mathrm{diff}, G}^{*}$, i.e. $\mathcal{I}\left[\mathrm{Cyl}_{\mathrm{diff}, G}^{*}\right]=\underline{\mathrm{Cyl}}_{\mathrm{diff}, G}^{*}$.

$(\subseteq)$ : Let $\eta \Psi \in \mathrm{Cyl}_{\mathrm{diff}, G}^{*}$ be given, so that $\Psi \in \mathrm{Cyl} \cap \mathcal{H}_{G}$, and in particular $\Psi \in \mathrm{Cyl}_{\gamma}$ for some $\gamma \in \Gamma$. By part (1) of lemma 4, there exists $\varphi \in$ Diff such that $\varphi \cdot \gamma \in \Gamma$. Then $\left(\varphi^{-1}\right)^{*} \Psi \in \underline{\mathrm{Cyl}} \cap \underline{\mathcal{H}}_{G}$, and we have $\mathcal{I} \eta \Psi=\mathcal{I} \eta\left(\varphi^{-1}\right)^{*} \Psi=\underline{\eta}\left(\varphi^{-1}\right)^{*} \Psi$, where lemma 5 was used in the second step. Thus, $\mathcal{I} \eta \Psi \in \underline{\mathrm{Cyl}}_{\mathrm{diff}, G}^{*}$.

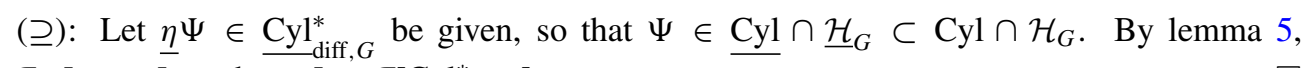
$\mathcal{I} \eta \Psi=\underline{\eta} \bar{\Psi}$ so that $\underline{\eta} \Psi \in \mathcal{I}\left[\mathrm{Cyl}_{\mathrm{diff}, G}^{*}\right]$.

\section{Equivalence of diffeomorphism-invariant operators, and equivalence of dynamics}

When constructing operators in plLQG, we propose one quantize in exactly the same way as in standard LQG, except that only piecewise linear edges should be used. For operators preserving Cyl, this general statement can be made precise as follows. Given an operator $\hat{O}_{\omega}$ in standard LQG, a corresponding operator is defined in plLQG iff $\hat{O}_{\omega}$ preserves Cyl, and in this case one defines the corresponding operator $\hat{O}_{p l}$ in plLQG to be $\left.\hat{O}_{\omega}\right|_{\text {Cyl }}$. An immediate consequence of this definition is

$$
\hat{O}_{p l}^{*} \circ \mathcal{I}=\mathcal{I} \circ \hat{O}_{\omega}^{*}
$$

Let an operator, in the analytic or piecewise linear framework, be called 'diffeomorphism invariant' if it is invariant under the group of generalized diffeomorphisms for the relevant framework. We then have the following.

Proposition 9. Given any diffeomorphism-invariant operator $\hat{O}_{\omega}$ preserving Cyl in standard $L Q G$, then $\hat{O}_{\omega}$ also preserves $\mathrm{Cyl}$. The corresponding piecewise linear operator $\hat{O}_{p l}$ is also diffeomorphism invariant, and $\hat{O}_{p l}$ and $\hat{O}_{\omega}$ are mapped into each other by the isomorphism $\left.\mathcal{I}\right|_{\text {Cyl }}{ }_{\text {diff }}$, that is,

$$
\hat{O}_{p l}^{*} \circ \mathcal{I}=\mathcal{I} \circ \hat{O}_{\omega}^{*} .
$$

Proof. Every diffeomorphism-invariant operator preserving Cyl is also graph preserving ${ }^{13}$. Therefore, $\hat{O}_{\omega}$ preserves also Cyl, so that $\hat{O}_{\mathrm{pl}}$ is defined and satisfies $(21)$, and is trivially Diff-invariant as Diff $\subset$ Diff.

\footnotetext{
${ }^{13}$ This can be seen as follows. Suppose $\hat{O}$ is Diff-invariant and preserves Cyl. Let $\Psi \in \mathrm{Cyl}_{\alpha}$ be given for some $\alpha$. As $\hat{O}$ preserves Cyl, $\hat{O} \Psi \in \mathrm{Cyl}_{\beta}$ for some $\beta$. From Diff-invariance, we have that for all $\varphi \in \operatorname{Diff}_{\alpha}$ (recall Diff $_{\alpha}$ is the subgroup of Diff preserving $\alpha$ ), $\hat{O} \Psi=U_{\varphi} \hat{O} U_{\varphi^{-1}} \Psi=U_{\varphi} \hat{O} \Psi$, so that $\hat{O} \Psi \in \mathrm{Cyl}_{\varphi \cdot \beta}$ for all $\varphi \in \operatorname{Diff}_{\alpha}$. Thus $\hat{O} \Psi \in \cap_{\varphi \in \operatorname{Diff}_{\alpha}} \mathrm{Cyl}_{\varphi \cdot \beta}$. But given any $\gamma, \gamma^{\prime} \in \Gamma, \mathrm{Cyl}_{\gamma} \cap \mathrm{Cyl}_{\gamma^{\prime}}=\mathrm{Cyl}_{\gamma \cap \gamma^{\prime}}$, so that $\hat{O} \Psi \in \mathrm{Cyl}_{\cap \varphi \in \operatorname{Diff}_{\alpha} \varphi \cdot \beta}$. The only edges of $\beta$ that survive in $\cap_{\varphi \in \operatorname{Diff}_{\alpha}} \varphi \cdot \beta$ are those that are also edges of $\alpha$, whence in fact $\hat{O} \Psi \in \mathrm{Cyl}_{\alpha}$, showing $\hat{O}$ is graph preserving.
} 
Of course there are also diffeomorphism-invariant operators that are not well defined on Cyl. The above proposition does not apply to them; rather they must be considered on a case by case basis. The master constraint is such an operator-it must be directly defined on $\mathrm{Cyl}_{\mathrm{diff}}^{*}$. We will later discuss the master constraint, after we have discussed the Hamiltonian constraint.

The Hamiltonian constraint [15] is rather unique because it has as its domain $\mathrm{Cyl}_{\mathrm{diff}}^{*}$, but does not map Cyl diff back into itself. It is defined as follows. For each lapse $N$, each $\epsilon \in\left[0, \epsilon_{0}\right]$ and each graph $\gamma$, one defines a regulated operator $\hat{H}(N)_{\gamma, \epsilon}$ on $\mathcal{H}_{\gamma}^{\prime}$ (see $[15,1]$ ). Piecing these together for all $\gamma$ gives, for each $\epsilon$, an operator $\hat{H}(N)_{\epsilon}$ on the kinematical Hilbert space

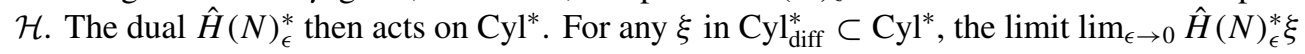
eventually becomes trivial [15], allowing us to define

$$
\hat{H}(N) \xi:=\lim _{\epsilon \rightarrow 0} \hat{H}(N)_{\epsilon}^{*} \xi,
$$

so that $\hat{H}(N)$ is well defined on $\mathrm{Cy}_{\text {diff }}^{*}$.

We will also need $\hat{H}(N)$ to be covariant with respect to the group of generalized diffeomorphisms Diff introduced in section 3. In defining the regulated operators $\hat{H}(N)_{\epsilon, \alpha}$, one uses the volume operator. As long as one uses the Rovelli-Smolin volume operator [10] (in contrast to [15] where one uses the Ashtekar-Lewandowski operator [11]), $\hat{H}(N)$ will be covariant with respect to Diff, that is, $\left(U_{\varphi}^{-1}\right)^{*} \circ \hat{H}(N) \circ U_{\varphi}^{*}=\hat{H}\left(\varphi^{*} N\right)$ for all $\varphi \in$ Diff, where $U_{\varphi}$ denotes the unitary action of $\varphi$ on $\mathcal{H}$ via pullback. Nevertheless, for general lapse $N$, as with the standard Hamiltonian constraint defined using the Ashtekar-Lewandowski volume operator, $\hat{H}(N)$ will map $\mathrm{Cyl}_{\text {diff }}^{*}$ out of itself due to $\hat{H}(N)$ not being diffeomorphism invariant. One can nevertheless define the solution to the Hamiltonian constraint to be the common kernel of the operators $\hat{H}(N)$ for all lapse $N$.

This construction can be repeated in the obvious way for plLQG: one need only ensure that the loops added by the regulated $\hat{H}(N)_{\gamma, \epsilon}$ are chosen to be piecewise linear. We do this, and then for $\gamma \in \underline{\Gamma}$, define $\underline{\hat{H}}(N)_{\gamma, \epsilon}:=\left.\hat{H}(N)_{\gamma, \epsilon}\right|_{\text {Cyl }}$. A construction exactly parallel to that above then goes through, giving us a family of operators $\underline{\hat{H}}(N)$, defined on $\underline{C y l}_{\text {diff }}^{*}$. Again as long as we use the Rovelli-Smolin strategy for defining the volume, $\underline{\hat{H}}(N)$ is diffeomorphism

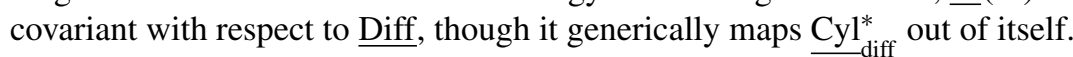

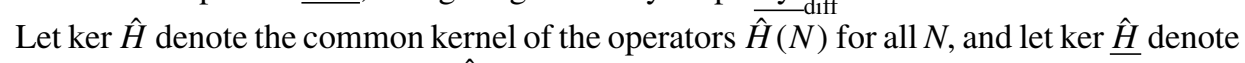
the common kernel of the operators $\underline{\hat{H}}(N)$ for all $N$. We have the following result.

Proposition 10. $\left.\mathcal{I}\right|_{\text {ker } \hat{H}}$ provides a unitary isomorphism from $\operatorname{ker} \hat{H}$ onto $\operatorname{ker} \underline{\hat{H}}$.

Proof. We first note that for $\Psi \in \mathrm{Cyl}_{\mathrm{diff}}^{*}, \Phi \in \mathrm{Cyl}$ and any lapse $N$, the following relation holds:

$$
\begin{aligned}
(\underline{\hat{H}}(N) \mathcal{I} \Psi|\Phi\rangle & :=\lim _{\epsilon \rightarrow 0}\left(\mathcal{I} \Psi\left|\underline{\hat{H}}(N)_{\epsilon} \Phi\right\rangle\right. \\
& =\lim _{\epsilon \rightarrow 0}\left(\Psi\left|\underline{\hat{H}}(N)_{\epsilon} \Phi\right\rangle=\lim _{\epsilon \rightarrow 0}\left(\Psi\left|\hat{H}(N)_{\epsilon} \Phi\right\rangle\right.\right. \\
& =(\hat{H}(N) \Psi|\Phi\rangle .
\end{aligned}
$$

From this we immediately see that if $\Psi \in \operatorname{ker} \hat{H}$, so that $\hat{H}(N) \Psi=0$ for all $N$, then $\hat{H}(N) \mathcal{I} \Psi=0$ for all $N$, so that $\mathcal{I} \Psi \in \operatorname{ker} \hat{\hat{H}}$, whence $\mathcal{I}[\operatorname{ker} \hat{H}] \subset \operatorname{ker} \underline{\hat{H}}$.

To prove the converse, let $\Theta \in \operatorname{ker} \underline{\hat{H}}$ be given. As $\underline{C y l}_{\text {diff }}^{*}$ is defined to be the domain of

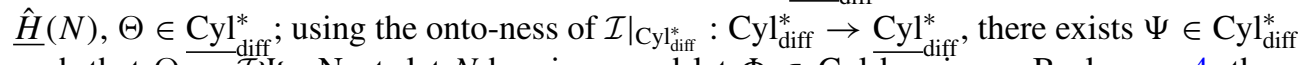
such that $\Theta=\mathcal{I} \Psi$. Next, let $N$ be given, and let $\Phi \in$ Cyl be given. By lemma 4 , there 
exists $\varphi \in$ Diff such that $\varphi^{*} \Phi \in$ Cyl. Using the Diff covariance of $\hat{H}(N)$ and then the Diff invariance of $(\Psi)$,

$$
\begin{aligned}
(\hat{H}(N) \Psi|\Phi\rangle & =\left(\left(U_{\varphi}^{-1}\right)^{*} \circ \hat{H}\left(\left(\varphi^{-1}\right)^{*} N\right) \circ\left(U_{\varphi}\right)^{*} \Psi|\Phi\rangle=\left(\hat{H}\left(\left(\varphi^{-1}\right)^{*} N\right) \Psi\left|U_{\varphi}^{-1}\right| \Phi\right\rangle\right. \\
& =\left(\hat{H}\left(\left(\varphi^{-1}\right)^{*} N\right) \Psi\left|\varphi^{*} \Phi\right\rangle .\right.
\end{aligned}
$$

Applying relation (24) to $\varphi^{*} \Phi$ and $\left(\varphi^{-1}\right)^{*} N$, and then using the fact that $\Theta=\mathcal{I} \Psi$ is in $\operatorname{ker} \hat{H}$, the last line above is seen to be zero. Thus, $(\hat{H}(N) \Psi|\Phi\rangle=0$ for all $\Phi \in \mathrm{Cyl}$ and all lapse $N$, proving $\Psi \in \operatorname{ker} \hat{H}$, so that $\Theta \in \mathcal{I}[\operatorname{ker} \hat{H}]$. This proves the containment $\operatorname{ker} \underline{\hat{H}} \subset \mathcal{I}[\operatorname{ker} \hat{H}]$, completing the proof that $\operatorname{ker} \underline{\hat{H}}=\mathcal{I}[\operatorname{ker} \hat{H}]$.

As already shown in theorem $6, \mathcal{I}$ is injective and unitary on $\mathrm{Cyl}_{\mathrm{diff}}^{*}$, so that it is also injective and unitary on $\operatorname{ker} \hat{H}$. Thus, $\left.\mathcal{I}\right|_{\text {ker } \hat{H}}: \operatorname{ker} \hat{H} \rightarrow \operatorname{ker} \underline{\hat{H}}$ provides a unitary isomorphism between $\operatorname{ker} \hat{H}$ and $\operatorname{ker} \underline{\hat{H}}$.

Finally, the physical Hilbert spaces of solutions to the diffeomorphism, Gauss and Hamiltonian constraint in LQG and plLQG are $\mathcal{H}_{\text {Phys }}:=\overline{\mathrm{Cyl}_{\text {diff }, G}^{*} \cap \operatorname{ker} \hat{H}}$ and $\underline{\mathcal{H}}_{\text {Phys }}:=$ $\overline{\mathrm{Cyl}}_{\mathrm{diff}, G}^{*} \cap \operatorname{ker} \underline{\hat{H}}$, respectively, where the closure denotes Cauchy completion. As the isomorphism $\left.\mathcal{I}\right|_{\text {Cyldiff }} ^{*}$ maps the inner product on $\mathrm{Cyl}_{\text {diff }}^{*}$ onto that on $\underline{\mathrm{Cyl}}_{\text {diff }}^{*}$, maps $\mathrm{Cyl}_{\mathrm{diff}, G}^{*}$ onto Cyl $_{\text {diff }, G}^{*}$ and maps $\operatorname{ker} \hat{H}$ onto $\operatorname{ker} \underline{\hat{H}}$, it is immediate that $\mathcal{I}$ provides a unitary isomorphism between these physical Hilbert spaces.

We now come to the master constraint. Let us review its construction in standard LQG from [16]. First, given a spatial point $v \in M$, let $N_{v}(x):=\delta_{v, x}$, a particular singular choice of lapse. The corresponding Hamiltonian constraint operator $\hat{H}_{v}:=\hat{H}\left(N_{v}\right)$ is nevertheless well defined [15]. We next recall the generalized spin-network functions $T_{\sigma}$, where $\sigma$ denotes the triple $(\gamma, \vec{j}, \vec{T})$ of a graph $\gamma \in \Gamma$, an assignment of a spin to each edge and an assignment of a tensor among representations to each vertex $[1,2]$. We require that all spin labels be non-trivial. Furthermore, as in, e.g., [1], for each possible set of representations incident at a vertex, we fixed a basis of the tensor space among the representations. Let $\mathcal{S}$ denote the space of all such triples $(\gamma, \vec{j}, \vec{T}) .\left\{T_{\sigma}\right\}_{\sigma \in \mathcal{S}}$ forms an orthonormal basis of Cyl and hence $\mathcal{H}$. Furthermore, Diff acts on $\mathcal{S}$, so that we may consider the Diff-equivalence class of an element $\sigma \in \mathcal{S}$, which we denote $[\sigma]_{\text {Diff. }}$. With these definitions made, we define a quadratic form $Q_{M}: \mathrm{Cyl}_{\mathrm{diff}}^{*} \times \mathrm{Cyl}_{\mathrm{diff}}^{*} \rightarrow \mathbb{C}$ by

$$
Q_{M}(\Phi, \Psi):=\sum_{[\sigma]_{\text {Diff }}} \eta_{[\sigma]_{\text {Diff }}} \sum_{v \in V(\gamma(\sigma))} \overline{\left(\hat{H}_{v} \Phi\left|T_{\sigma}\right\rangle\right.}\left(\hat{H}_{v} \Psi\left|T_{\sigma}\right\rangle,\right.
$$

where $\eta_{[\sigma]_{\text {Diff }}}:=1 /\left|G S_{\gamma(\sigma)}\right|$ are the coefficients appearing in the last expression in (14) for the diffeomorphism constraint rigging map, and where $V(\gamma(\sigma))$ denotes the set of vertices in $\gamma(\sigma) .{ }^{14} Q_{M}(\cdot, \cdot)$ then determines the master constraint $\hat{M}$ uniquely via [16]

$$
\hat{M} \Phi:=\sum_{x \in I} Q_{M}\left(B_{x}, \Phi\right) B_{x}
$$

where $\left\{B_{x}\right\}_{x \in I}$ is any orthonormal basis of Cyl ${ }_{\text {diff }}^{*}$.

A construction parallel to the above goes through in the plLQG case. Let $\underline{\mathcal{S}}$ denote the

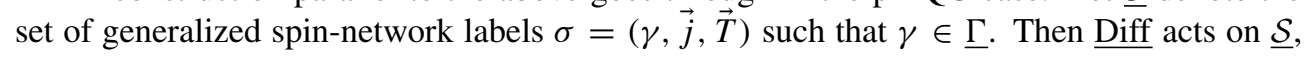

\footnotetext{
${ }^{14}$ In [16], $\eta_{[\sigma]_{\text {Diff }}}$ are a set of constants parametrizing an ambiguity in the definition of the rigging map discussed in the original work [18]. Here, as earlier in this paper, we are taking a natural resolution to this ambiguity suggested in [1], leading to the specific values of $\eta_{[\sigma]_{\text {Diff }}}$ given above.
} 
so that for each $\sigma \in \underline{\text { Diff, }}$, one can define an equivalence class $[\sigma]_{\text {Diff. }}$ The quadratic form for the piecewise linear framework is then

$$
Q_{\underline{M}}(\Phi, \Psi):=\sum_{[\sigma]_{\underline{\text { Diff }}}} \eta_{[\sigma]_{\text {Diff }}} \sum_{v \in V(\gamma(\sigma))} \overline{\left(\underline{\hat{H}}_{v} \Phi\left|T_{\sigma}\right\rangle\right.}\left(\underline{\hat{H}}_{v} \Psi\left|T_{\sigma}\right\rangle\right.
$$

where $\Phi, \Psi \in \mathrm{Cyl}_{\text {diff }}^{*}$, and where $\eta_{[\sigma]_{\text {Diff }}}=1 /\left|G \underline{S}_{\gamma(\sigma)}\right|$ are the coefficients in the plLQG rigging map (9). The master constraint is then

$$
\underline{\hat{M}} \Phi:=\sum_{x \in I} Q_{\underline{M}}\left(\underline{B}_{x}, \Phi\right) \underline{B}_{x}
$$

where $\left\{\underline{B}_{x}\right\}_{x \in I}$ is any orthonormal basis of $\underline{\mathrm{Cyl}}_{\mathrm{diff}}^{*}$.

Proposition 11. $\hat{M}$ is mapped into $\underline{\hat{M}}$ by the isomorphism $\left.\mathcal{I}\right|_{\text {Cyl }{ }_{\mathrm{diff}}^{*}}$.

Proof. In each case the master constraint is determined from the quadratic form and inner product on diffeomorphism invariant states in the same way. To prove equivalence of the master constraints, it is thus sufficient to prove equivalence of the quadratic forms; that is, we want to show $Q_{\underline{M}}(\mathcal{I} \Phi, \mathcal{I} \Psi)=Q_{M}(\Phi, \Psi)$ for all $\Phi, \Psi \in \mathrm{Cyl}_{\mathrm{diff}}^{*}$ :

$$
\begin{aligned}
& Q_{\underline{M}}(\mathcal{I} \Phi, \mathcal{I} \Psi):=\sum_{[\sigma]_{\underline{\text { Diff }}}} \eta_{[\sigma]_{\text {Diff }}} \sum_{v \in V(\gamma(\sigma))} \overline{\left(\underline{\hat{H}}_{v} \mathcal{I} \Phi\left|T_{\sigma}\right\rangle\right.}\left(\underline{\hat{H}}_{v} \mathcal{I} \Psi\left|T_{\sigma}\right\rangle\right. \\
& =\sum_{[\sigma]_{\underline{\text { Diff }}}} \eta_{[\sigma]_{\text {Diff }}} \sum_{v \in V(\gamma(\sigma))} \lim _{\epsilon, \epsilon^{\prime} \rightarrow \infty} \overline{\left(\mathcal{I} \Phi\left|\underline{\hat{H}}_{v, \epsilon} T_{\sigma}\right\rangle\right.}\left(\mathcal{I} \Psi\left|\underline{\hat{H}}_{v, \epsilon} T_{\sigma}\right\rangle\right. \\
& =\sum_{[\sigma]_{\text {Diff }}} \eta_{[\sigma]_{\text {Diff }}} \sum_{v \in V(\gamma(\sigma))} \lim _{\epsilon, \epsilon^{\prime} \rightarrow \infty} \overline{\left(\Phi\left|\hat{H}_{v, \epsilon} T_{\sigma}\right\rangle\right.}\left(\Psi\left|\hat{H}_{v, \epsilon} T_{\sigma}\right\rangle\right. \\
& =\sum_{[\sigma]_{\text {Diff }}} \eta_{[\sigma]_{\underline{\text { Diff }}}} \sum_{v \in V(\gamma(\sigma))} \overline{\left(\hat{H}_{v} \Phi\left|T_{\sigma}\right\rangle\right.}\left(\hat{H}_{v} \Psi\left|T_{\sigma}\right\rangle\right.
\end{aligned}
$$

where, in the third equality, we have used the definition of $\mathcal{I}$ and that $\underline{\hat{H}}_{v, \epsilon}=\left.\hat{H}_{v, \epsilon}\right|_{\text {Cyl }}$. Now, the

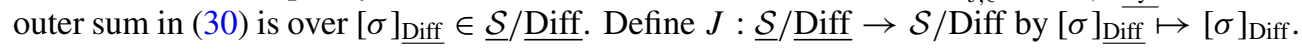
$J$ is well defined due to $\underline{\text { Diff }} \subset$ Diff. Using lemma 2 , one shows that it is $1-1$, and using lemma 4 one sees that it is onto. (Details: exercise for the reader.) Furthermore, as $\sigma \in \underline{\mathcal{S}}$, $\gamma(\sigma) \in \underline{\Gamma}$, so that from lemma $3,|\underline{G S} \underline{\gamma(\sigma)}|=\left|G S_{\gamma(\sigma)}\right|$, and we have $\eta_{[\sigma]_{\text {Diff }}}=\eta_{[\sigma]_{\text {Diff }}}$. Using the isomorphism $J$ to replace $[\sigma]_{\text {Diff }}$ with $[\sigma]_{\text {Diff }}$ in (30), we obtain

$$
Q_{\underline{M}}(\mathcal{I} \Phi, \mathcal{I} \Psi)=Q_{M}(\Phi, \Psi) .
$$

Lastly, because $\left.\mathcal{I}\right|_{\mathrm{Cy}_{\mathrm{diff}}^{*}}$ maps the master constraint $\hat{M}$ onto $\underline{\hat{M}}$, and $\mathrm{Cyl}_{\mathrm{diff}, G}^{*}$ onto $\underline{\mathrm{Cyl}}_{\mathrm{diff}, G}^{*}$, $\left.\mathcal{I}\right|_{\mathrm{Cy}_{\mathrm{diff}, G}^{*}}$ will map $\left.\hat{M}\right|_{\mathrm{Cy} l_{\mathrm{diff}, G}^{*}}$ onto $\left.\underline{\hat{M}}\right|_{\left.{ }_{\mathrm{Cyl}}\right|_{\mathrm{diff}, G} ^{*}}$, so that the master constraint dynamics are also equivalent after solving both the diffeomorphism and Gauss constraints ${ }^{15}$.

The above results show that not only are the diffeomorphism-invariant Hilbert spaces in LQG and plLQG unitarily isomorphic, but the dynamics (whether defined with master or Hamiltonian constraint) are isomorphic as well, so that the two frameworks are truly equivalent.

${ }^{15}$ In [16], the master constraint is in fact constructed directly on $\mathrm{Cyl}_{\mathrm{diff}, G}^{*}$. 


\section{Exact embedding of LQC into piecewise linear LQG}

In this section, we will consider an application of the above constructions to cosmology. The restriction of the topology and piecewise linear structure of $M$ to be that of $\mathbb{R}^{3}$ is here no longer for simplicity, but is implied by homogeneity and isotropy.

As mentioned briefly in the introduction, the restriction, to the homogeneous isotropic sector, of the configuration algebra of LQG $(\mathrm{Cyl})$ is not equal to the configuration algebra of LQC (the almost periodic functions-see the definition below). As a consequence, the paper [5] was not able to construct an embedding of LQC states into the usual space of distributional states in LQG, Cyl*. Instead, an embedding of LQC states into what was at the time a more unusual space, $\mathrm{Cyl}^{*}$, had to be defined. A possible physical meaning for $\mathrm{Cyl}^{*}$ was suggested in [5]. Nevertheless, it was not clear how to use Cyl ${ }^{*}$ for the next step in the program of $[4,5]$. Specifically, the next step was to group average the kinematical embeddings to obtain embeddings into LQG at the diffeomorphism-invariant level. Note one must construct embeddings into LQG at the diffeomorphism-invariant level if one hopes to exactly relate the Hamiltonian constraints in LQC and LQG in any way, as the latter is defined only on diffeomorphism-invariant states. To accomplish the construction of the diffeomorphism-invariant embeddings, two issues needed to be addressed [5].

(1) The group of piecewise analytic diffeomorphisms did not even act on Cyl ${ }^{*}$, so that one could not even write down a formal expression for group averaging the kinematical embeddings.

(2) Once one is able to write down a formal group averaging, one would need to regulate the integral over diffeomorphisms in some way.

It is in this first step that the use of $\mathrm{Cyl}^{*}$ seemed to prevent further progress.

In the construction of plLQG, $\overline{\mathrm{Cyl}^{*}}$ also appears, but this time as the space of distributional states for a completely parallel framework for loop quantum gravity, which, as was proven above, is equivalent to the standard one at the diffeomorphism-invariant level. Furthermore, the space of 'piecewise linear generalized diffeomorphisms' acts on $\mathrm{Cyl}^{*}$, so that one can now formally write down the group averaging of the embeddings, providing an expression for the embedding into the space of diffeomorphism-invariant states. Because of the isomorphism between plLQG and LQG at the diffeomorphism-invariant level, this is also a formal expression for the embedding into the space of diffeomorphism-invariant states in standard LQG. That is, the first obstruction listed above no longer exists. Because the embeddings of [5] were a motivation for the present work, we briefly review them here; we then end the section with the new expressions for the diffeomorphism-invariant embeddings.

First we recall some necessary structures from loop quantum cosmology (LQC). As in [25], we take the classical configuration space for homogeneous, isotropic cosmology to be the space of homogeneous, isotropic connections, but in the gauge-fixed sense defined in [5]; we denote this space by $\mathcal{A}_{S}$. By picking a reference connection $\AA_{a}^{i} \in \mathcal{A}_{S}$, all other connections in $\mathcal{A}_{S}$ are related to $\AA_{a}^{i}$ by scaling. Thus, if we define $r: \mathbb{R} \rightarrow \mathcal{A}_{S} \subset \mathcal{A}$ by

$$
r: c \mapsto c \AA_{a}^{i},
$$

$r$ provides an isomorphism of $\mathbb{R}$ with $\mathcal{A}_{S}$. States in LQC are then functions on $\mathbb{R} \cong \mathcal{A}_{S}$. The basic space of 'nice' states in LQC (and one of the sources of the unique character of LQC) is the space of almost periodic functions; following [25], we denote this $\mathrm{Cyl}_{S} \cdot \mathrm{Cyl}_{S}^{*}$ is the space of distributional states.

The kinematical and gauge-invariant embeddings of [5] are then defined as follows. The 'c' embedding $\iota_{c}: \mathrm{Cyl}_{S}^{*} \rightarrow \mathrm{Cyl}^{*}$ is defined by

$$
\left(\iota_{c} \psi|\Phi\rangle:=\overline{(\psi \mid} r^{*} \Phi\right\rangle \text {. }
$$


From $\iota_{c}$, one constructs the ' $b$ ' embeddings. To remind the reader from $[4,5]$, the ' $b$ ' embedding is built using coherent states, the idea being to use the freedom in the choice of coherent states to adapt the embedding to be approximately preserved by the dynamics. In $[4,5]$, complexifier coherent states are used; in complexifier coherent states, the freedom in choosing the family of coherent states is parametrized by a choice of complexifier [26]. To introduce the complexifiers, first let $X_{S}$ and $X$ denote the classical phase space of the reduced and full theories, respectively. Then let $C_{S}: X_{S} \rightarrow \mathbb{R}^{+}, C: X \rightarrow \mathbb{R}^{+}$be any two (pure momentum) complexifiers [26]. Let $\hat{C}_{S}$ and $\hat{C}$ denote their respective quantizations in the reduced and full quantum theories. For brevity, we give only the final expression for the corresponding ' $b$ ' embedding $\iota_{b}: \mathrm{Cyl}_{S}^{*} \rightarrow \mathrm{Cyl}^{*}$. It is given by [5]

$$
\left(\iota_{b} \psi|\Phi\rangle:=\left(\psi\left|e^{\hat{C}_{S}} \circ r^{*} \circ e^{-\hat{C}}\right| \Phi\right\rangle .\right.
$$

The Gauss-gauge invariant versions of these embeddings are $\iota_{c}^{G}:=P_{G}^{*} \circ \iota_{c}$ and $\iota_{b}^{G}:=P_{G}^{*} \circ \iota_{b}$, where $P_{G}: \mathrm{Cyl} \rightarrow \mathrm{Cyl}$ denotes the projector onto gauge-invariant states. For the motivation behind these definitions and their nice properties, we refer the reader to the original papers $[4,5]$.

Now we come to the formal expression for the embedding into diffeomorphism-invariant states made possible by the new piecewise linear LQG framework introduced in this paper. The diffeomorphism-invariant embedding $\iota_{c}^{\text {Diff }}: \mathrm{Cyl}_{S}^{*} \rightarrow \underline{\mathrm{Cy}}_{\text {diff }}^{*}$ has the formal expression

$$
\begin{aligned}
\left(\iota_{c}^{\text {Diff }} \psi|\Phi\rangle\right. & :=\left(\int_{\varphi \in \underline{\text { Diff }}} \mathcal{D} \varphi\left(U_{\varphi}^{*} \iota_{c}^{G} \psi \mid\right)|\Phi\rangle\right. \\
& =\int_{\varphi \in \text { Diff }} \mathcal{D} \varphi\left(\iota_{c}^{G} \psi\left|U_{\varphi}\right| \Phi\right\rangle \\
& =\int_{\varphi \in \underline{\text { Diff }}} \mathcal{D} \varphi\left(\psi\left|r^{*} \circ P_{G} \circ U_{\varphi}\right| \Phi\right\rangle .
\end{aligned}
$$

The formal expression for the diffeomorphism-invariant ' $b$ ' embedding $\iota_{b}^{\text {Diff }}: \mathrm{Cyl}_{S}^{*} \rightarrow$ Cyl $_{\text {diff }}^{*}$ is then

$$
\left(\iota_{b}^{\text {Diff }} \psi|\Phi\rangle=\int_{\varphi \in \underline{\text { Diff }}} \mathcal{D} \varphi\left(\psi\left|e^{\hat{C}_{S}} \circ r^{*} \circ e^{-\hat{C}} \circ P_{G} \circ U_{\varphi}\right| \Phi\right\rangle .\right.
$$

If $\hat{C}$ and $\hat{C}_{S}$ are gauge and diffeomorphism invariant, this reduces to $\iota_{b}^{\text {Diff }}=e^{-\hat{C}^{*}} \circ \iota_{c}^{\text {Diff }} \circ e^{\hat{C}_{S}^{*}}$. Composing (35) and (36) with the isomorphism $\underline{\mathrm{Cyl}}_{\mathrm{diff}}^{*} \leftrightarrow \mathrm{Cyl}_{\mathrm{diff}}^{*}$ defined in section 3 then provides us with the formal expression for the 'c' embedding into $\mathrm{Cyl}_{\text {diff }}^{*}$, and for the ' $b$ ' embeddings into Cyl diff $^{*}$ The use of Diff instead of Diff not only has allowed us to write these expressions, but the fact that Diff is so much smaller than Diff makes it more likely that they can be regularized.

\section{Discussion}

The kinematics of LQG are usually formulated in terms of the piecewise analytic category. We have shown that the piecewise analytic category is not essential, and can be replaced with something as simple as the piecewise linear category, giving rise to what we have called piecewise linear LQG (plLQG). We have shown that piecewise linear LQG is fully equivalent to standard LQG at the diffeomorphism-invariant level, both in terms of Hilbert space structure and dynamics, as long as one makes a choice of generalized diffeomorphism group such as advocated in $[9,14,22]$. 
We have additionally seen that LQC is exactly embeddable into plLQG. This suggests that the non-embeddability result of [6] may be somewhat of a red herring: it appears relevant at the kinematical level, but this relevance seems to evaporate at the diffeomorphism-invariant level. For, plLQG circumvents the non-embeddability result of [6], and is yet fully equivalent to LQG at the diffeomorphism-invariant level. This is what has now allowed us to at least write down formal expressions for embeddings of LQC into LQG at the diffeomorphism-invariant level, of the type motivated in $[4,5]$. These expressions were given in section $5 .{ }^{16}$ Of course it still remains to regulate these expressions in some way.

A side effect of the choice of generalized diffeomorphisms is that one is obliged to use the Rovelli-Smolin volume operator [10] rather than the Ashtekar-Lewandowski volume operator [11] in defining the dynamics. Arguments are present in the literature both in favor of the Rovelli-Smolin volume operator $[9,22]$ and in favor of the Ashtekar-Lewandowski volume operator [27]. ${ }^{17}$ The present research does not directly add to this discussion; the only statements the present work can make on this topic are (1) if one uses the piecewise linear category, one is forced to use the Rovelli-Smolin volume operator in the dynamics, and (2) the exact embedding in this paper of LQC into LQG is only possible if the Rovelli-Smolin volume operator is used.

We close with some remarks regarding the similarities of piecewise linear LQG to the framework underlying the construction of spinfoams. As argued, for example, in [8], the classical theory underlying spinfoams is a certain discrete theory based on piecewise flat geometries. Furthermore, as touched upon in appendix B of [8], in order for the discrete variables to fully describe the piecewise flat geometry, one is implicitly assuming a given linear structure on each patch. Thus, one is actually assuming a piecewise linear structure of spacetime. As seen in this paper, the use of piecewise linear structures naturally leads to the use of simplicial complexes, and simplicial complexes are central in the classical discrete theory underlying spinfoams. Whether the relation between plLQG and spinfoams goes beyond these cursory remarks is not clear, and would be interesting to investigate.

\section{Acknowledgments}

The author thanks Tim Koslowski for stimulating exchanges and Andrew Ranicki for encouraging him to study simplicial complexes in more depth. Thomas Thiemann, Bianca

\footnotetext{
${ }^{16}$ As a side note, it may also be possible that there is another way to relate LQG to cosmology other than via the piecewise linear framework presented here. For, as pointed out by Koslowski [28], it appears that, given any analytic edge $e$, the holonomy along $e$ as a function of the symmetric connection $A_{S}=r(c)=c \AA_{a}^{i}$ can be decomposed into an almost periodic part [25] and a part vanishing as $c$ approaches infinity. If true, it is not hard to see that this decomposition must be unique, as there are no almost periodic functions that vanish at infinity. This would then allow one to construct a projector $P_{\mathrm{ap}}: r^{*}[\mathrm{Cyl}] \rightarrow \mathrm{Cyl}_{S}$ that projects out the almost periodic part. The projector could then be used to construct embeddings $\iota_{c}$ and $\iota_{b}$ of LQC directly into Cyl*: $\left(\iota_{c} \psi|\Phi\rangle:=\left(\psi\left|P_{\mathrm{ap}} r^{*}\right| \Phi\right\rangle\right.$, and then $\iota_{b}:=e^{-\hat{C}^{*}} \circ \iota_{c} \circ e^{\hat{C}_{S}^{*}}$. These embeddings would again satisfy the physical intertwining criterion used in [5]. As the codomain of such embeddings would be directly $\mathrm{Cyl}^{*}$, and Diff acts on Cyl*, one would then be able to directly write down a formal expression for ' $c$ ' and ' $b$ ' embeddings into diffeomorphism-invariant states, similar to that in section 5 of this paper. One could then check whether the resulting formal embedding is equivalent to the one given in this paper. Of course, the resulting embedding would also have to be regularized.

${ }^{17}$ Although [27] says that the Rovelli-Smolin volume operator leads to an 'inconsistent' quantization, one needs to be careful as to the meaning of 'inconsistent' here. It does not mean that there is a contradiction in the resulting quantum theory. The work [27] shows that if one uses the Rovelli-Smolin volume operator, then a certain classical relation, when regularized in a certain specific way, leads to an equation that does not hold in the quantum theory. But it is well known that one cannot expect all classical relations to hold in the quantum theory; this is especially true if a specific choice of regularization is involved. Furthermore, the work by Rovelli, Fairbairn [22] and Koslowski [9] give independent arguments in favor of the Rovelli-Smolin volume operator that seem to many to be as convincing as those of [27].
} 
Dittrich and the referees are also thanked for helpful remarks on a prior draft. This work was supported in part by the Alexander von Humboldt foundation of Germany and by NSF grant OISE-0601844.

\section{Appendix A. General definitions and proof of a lemma}

In this appendix, we first give the general definition of a piecewise linear structure, and the associated more general versions of definitions given in the main text. With these more general versions of the definitions, in fact all arguments in the present paper go through unchanged (except in section 5, where the topology and piecewise linear structure are fixed by the desired application). We then prove a lemma that is used in the main text, using these more general definitions.

\section{A.1. General definitions}

Although it is sufficient to use simplices to define the notion of a general piecewise linear structure, it will be very useful for the next section to use cells. A cell in $\mathbb{R}^{n}$ is defined in the same way as a simplex, except one does not require independence of the vertices. That is, a cell $\tilde{C} \subset \mathbb{R}^{n}$ is the convex hull of some finite set of points $v_{1}, \ldots, v_{m}$ :

$$
\tilde{C}=\left\{\sum_{i} \lambda_{i} v_{i} \text { such that } \sum_{i} \lambda_{i}=1 \text { and } \lambda_{i}>0 \text { for all } i\right\} .
$$

Given a cell $\tilde{C}$ and an affine subspace $P \subset \mathbb{R}^{n}$ such that $C \backslash P$ is connected, we call $\tilde{A}:=P \cap \tilde{C}$ a face of $\tilde{C}$, and we write $\tilde{A} \leqslant \tilde{C}$. For $\tilde{C}$ three dimensional, the vertices, edges, faces of $\tilde{C}$ in the usual sense, as well $\tilde{C}$ itself, are faces of $\tilde{C}$.

Consider next a differentiable $n$-manifold $\Sigma$. If a subset $C \subset \Sigma$ admits a coordinate chart $\varphi_{C}: \mathcal{O} \subset \Sigma \rightarrow \mathcal{O}^{\prime} \subset \mathbb{R}^{n}$ mapping $C$ onto a cell in $\mathbb{R}^{n}$, we call $C$ a differentiable cell. Given any face $\tilde{A}$ of $\varphi_{C}[C], A:=\varphi_{C}^{-1}[\tilde{A}]$ is also differentiable cell, we call it a face of $C$ and we write $A \leqslant C$. Define a differentiable cell complex to be a (locally finite) collection of differentiable cells $K$ satisfying

(1) if $C \in K$ and $B$ is a face of $C$, then $B \in K$ and;

(2) if $B, C \in K$, then $B \cap C$ is a face of $B$ and $C$.

One way to construct a general piecewise linear structure on $\Sigma$ is then the following. Choose a differentiable cell complex $K$ covering all of $\Sigma$. For each $n$-dimensional cell $C \in K$, choose one of the possible coordinate charts $\varphi_{C}$ mapping $C$ onto a cell in $\mathbb{R}^{n}$, and let $\partial_{a}$ denote, within $C$, the coordinate derivative associated with this coordinate chart. Piecing these connections together yields a connection $\partial_{a}$ on $\Sigma$ that is flat everywhere except possibly on submanifolds of dimension not greater than $n-2$; it is this connection that defines the piecewise linear structure on $\Sigma$. A manifold equipped with a piecewise linear structure we call a piecewise linear manifold. Any division of $\Sigma$ into a cell complex that can also be used to construct this same $\partial_{a}$ we call a cellular decomposition compatible with the piecewise linear structure.

More generally, any subset $C$ of $\Sigma$ admitting a coordinate chart $\varphi_{C}$ mapping $C$ onto a cell in $\mathbb{R}^{n}$ and mapping $\partial_{a}$ into the standard flat connection on $\mathbb{R}^{n}$ we call a linear cell, or simply a cell, and we call $\varphi_{C}$ a local linear chart. A differentiable cell complex all of whose cells are linear we call a linear cell complex, or simply a cell complex. In the case when the cells are in particular simplices, this generalizes the notion of simplicial complex to an arbitrary piecewise linear manifold. 
Given two general piecewise linear $n$-manifolds $M$ and $N$, the definition of a piecewise linear homeomorphism $F: M \rightarrow N$ is the same as in the main text, with condition (6) for linearity in each $n$-simplex formulated as

$$
F \circ \varphi^{-1}\left[\sum_{i=0}^{n} t_{i} \varphi\left(v_{i}\right)\right]=\left(\varphi^{\prime}\right)^{-1}\left[\sum_{i=0}^{n} t_{i} \varphi^{\prime}\left(F\left(v_{i}\right)\right)\right]
$$

where $\varphi$ is any local linear chart for the $n$-simplex in $M$, and $\varphi^{\prime}$ is any local linear chart for the $n$-simplex in $N$.

The rest of the definitions in the main text, in terms of the above basic notions, remain unchanged. We now come to the proof of the lemma used in the main text.

\section{A.2. Existence of triangulation compatible with a graph}

The lemma proved here is needed in section 3 for demonstrating the unitary isomorphism between the diffeomorphism-invariant Hilbert spaces of plLQG and LQG. For the purposes of this appendix, we remind the reader, from section 3, that a 1-complex $X$ is said to be compatible with a piecewise linear graph $\gamma$ if $|X|$ is equal to the image of $\gamma$.

Given a cell complex $K$, we define $|K|:=\cup_{A \in K} A$ as the polyhedron underlying $K$. Given two cell complexes $K$ and $L, K$ is said to be a subdivision of $L$ if $|K|=|L|$ and every cell in $K$ is contained in a cell of $L$.

Next, given two points $x, y$ in $\mathbb{R}^{n}$, let $x y$ denote the line segment between them. Given a cell $\tilde{A}$ in $\mathbb{R}^{n}$, and a point $p$ not in the plane determined by $\tilde{A}$, define the cone with vertex $x$ and base $\tilde{A}$, denoted $x \tilde{A}$, by $x \tilde{A}:=\cup_{y \in \tilde{A}}(x y)$. Given now a cell $C$ in $M$, a face $A$ of $C$ and a point $p \in C$ not in $A$, letting $\varphi_{C}$ denote the local linear chart on $C$, we define the cone $p A$ by

$$
p A:=\varphi_{C}^{-1}\left[\varphi_{C}(p) \varphi_{C}(A)\right] .
$$

A subdivision $K$ of $L$ is then said to be obtained by starring at a point $p$ if $K$ is obtained from $L$ by replacing each cell $C \in L$ containing $p$ by the collection of cells $\{p F \mid F \leqslant C, a \notin F\}$ (see $\mathrm{p} 15$ of [29]).

With these preliminaries out of the way, we come to the lemma.

Lemma 12. Given any piecewise linear manifold $M$ and a piecewise linear graph $\gamma$ thereon, there exists a triangulation $K$ of $M$ containing a one-dimensional subcomplex $K_{1}$ compatible with $\gamma$.

Proof. Let $K_{0}$ be any of the cellular decompositions of $M$ compatible with its piecewise linear structure. Let $\tilde{X}$ be the minimal 1-complex compatible with $\gamma$ : that is, break up each edge of $\gamma$ into its straight parts, and define $\tilde{X}$ to contain all of these straight parts and their end points. $\tilde{X}$ is then finite. Define

$$
X:=\tilde{X} \cap K_{0}:=\left\{A \cap B \mid A \in \tilde{X}, B \in K_{0}\right\} .
$$

Because $\tilde{X}$ consists in a finite number of compact simplices, $|\tilde{X}|$ is compact. This, together with the local finiteness of $K_{0}$, implies that $X$ is also finite. For each 1-simplex $e$ in $X$, let $e_{1}, e_{2}$ denote the end points. Construct a cell complex $K_{e_{1}}$ by starring $K_{0}$ at the point $e_{1}$. Then construct $K_{e}$ by starring $K_{e_{1}}$ at $e_{2}$. Because $X$ is obtained via an intersection with $K_{0}$, every 1-simplex $e \in X$ is contained entirely in a single simplex of $K_{0}$. As a consequence, one can deduce that every cell of $K_{e_{1}}$ containing $e_{2}$ possesses $e_{1}$ as a vertex. The construction of $K_{e}$ using the starring procedure then guarantees that $e_{2} e_{1}=e$ will belong to $K_{e}$. Next, take the repeated intersection of the cell complexes $K_{e}$ so constructed:

$$
Q:=\left\{\cap_{e \in X} A_{e} \mid\left\{A_{e} \in K_{e}\right\}_{e \in X}\right\} .
$$


Noting example 2.8(5) of [29], this is again a cell complex ${ }^{18}$. Furthermore, $Q$ is a subdivision of each cell complex $K_{e}$. It therefore contains a subdivision $\tilde{e}$ of each $e$. Taking the union of these subdivisions $\tilde{e}$ provides a 1-complex $K_{1}$ that is a subcomplex of $Q$, and that is compatible with $\gamma$. Furthermore, from proposition 2.9 of [29], $Q$ can be subdivided further to obtain a simplicial complex $K$, without adding any vertices, so that $K_{1}$ is again a subcomplex of $K$. This gives a triangulation $K$ of $M$ containing a one-dimensional subcomplex compatible with $\gamma$, as desired.

\section{References}

[1] Ashtekar A and Lewandowski J 2004 Background independent quantum gravity: a status report Class. Quantum Grav. 21 R53

[2] Rovelli C 2004 Quantum Gravity (Cambridge: Cambridge University Press)

Smolin L 2004 An invitation to loop quantum gravity Quantum Theory and Symmetries (Cincinnati, OH, 10-14 September 2003) pp 655-82 (hep-th/0408048)

Thiemann T 2003 Lectures on loop quantum gravity Lect. Notes Phys. 631 41-135 (gr-qc/0210094)

Thiemann T 2007 Modern Canonical Quantum General Relativity (Cambridge: Cambridge University Press)

Ashtekar A 2007 An introduction to loop quantum gravity through cosmology Nuovo Cimento B 122 135-55

[3] Rovelli C and Smolin L 1988 Knot theory and quantum gravity Phys. Rev. Lett. 61 1155-8

Rovelli C and Smolin L 1990 Loop representation for quantum general relativity Nucl. Phys. B 331 80-152

Ashtekar A and Isham C J 1992 Representations of the holonomy algebras of gravity and non-Abelian gauge theories Class. Quantum Grav. 9 1433-68

Ashtekar A and Lewandowski J 1993 Representation theory of analytic holonomy C* algebras Knots and Quantum Gravity ed J Baez (Oxford: Oxford University Press)

Rovelli C and Smolin L 1995 Spin networks and quantum gravity Phys. Rev. D 52 5743-59

[4] Engle J 2006 Quantum field theory and its symmetry reduction Class. Quantum Grav. 23 2861-94

[5] Engle J 2007 Relating loop quantum cosmology to loop quantum gravity: symmetric sectors and embeddings Class. Quantum Grav. 24 5777-802

[6] Brunnemann J and Fleischhack C 2007 On the configuration spaces of homogeneous loop quantum cosmology and loop quantum gravity arXiv:0709.1621

[7] Reisenberger M P and Rovelli C 1997 'Sum over surfaces' form of loop quantum gravity Phys. Rev. D 56 3490-508

Reisenberger M P 1997 A lattice worldsheet sum for 4-d euclidean GR arXiv:gr-qc/9711052

Baez J 1998 Spin foam models Class. Quantum Grav. 15 1827-58

Oriti D 2001 Spacetime geometry from algebra: spin foam models for non-perturbative quantum gravity Rep. Prog. Phys. 64 1703-57

Perez A 2003 Spin-foam models for quantum gravity Class. Quantam Grav. 20 R43-104

Freidel L 2005 Group field theory: an overview Int. J. Theor. Phys. 44 1769-83

[8] Engle J, Pereira R and Rovelli C 2008 Flipped spinfoam vertex and loop gravity Nucl. Phys. B 789 251-90

[9] Koslowski T 2006 Physical diffeomorphisms in loop quantum gravity arXiv:gr-qc/0610017

[10] Rovelli C and Smolin L 1995 Discreteness of area and volume in quantum gravity Nucl. Phys. B 442 593-622

[11] Ashtekar A and Lewandowski J 1998 Quantum theory of geometry II: volume operators Adv. Theor. Math. Phys. $1388-429$

[12] Lewandowski J 1997 Volume and quantization Class. Quantum Grav. 14 71-6

[13] Zapata J 1997 A combinatorial approach to diffeomorphism invariant quantum gauge theories J. Math. Phys. 38 5663-81

[14] Zapata J 1998 Combinatorial space from loop quantum gravity Gen. Rel. Grav. 30 1229-45

[15] Thiemann T 1998 Quantum spin dynamics (QSD) Class. Quantum Grav. 15 839-73

[16] Thiemann T 2006 Quantum spin dynamics VIII. The master constraint Class. Quantum Grav. 23 2249-66

[17] Ashtekar A and Lewandowski J 1996 Quantum theory of geometry: I. Area operators Class. Quantum Grav. 14 A55-82

[18] Ashtekar A, Lewandowski J, Marolf D, Mourão J and Thiemann T 1995 Quantization of diffeomorphism invariant theories of connections with local degrees of freedom J. Math. Phys. 36 6456-93

[19] Munkres J 1984 Elements of Algebraic Topology (Cambridge, MA: Perseus)

${ }^{18}$ The example here cited considers finite complexes; however, it is straightforward to see that it extends to locally finite complexes. 
[20] Fleischhack C 2006 Representations of the Weyl algebra in quantum geometry arXiv:math-ph/0407006

[21] Hardt R M 1975 Stratification of real analytic mappings and images Invent. Math. 28 193-208

[22] Fairbairn W and Rovelli C 2004 Separable Hilbert space in loop quantum gravity J. Math. Phys. 45 2802-14

[23] Alexander J W 1929 Combinatorial analysis situs Trans. Am. Math. Soc. 28 301-29

[24] Brown E 1969 The hauptvermutung for 3-complexes Trans. Am. Math. Soc. $144173-96$

[25] Ashtekar A, Bojowald M and Lewandowski J 2003 Mathematical structure of loop quantum cosmology $A d v$. Theor. Math. Phys. 7 233-68

[26] Thiemann T 2006 Complexifier coherent states for quantum general relativity Class. Quantum Grav. $232063-118$

[27] Giesel K and Thiemann T 2006 Consistency check on volume and triad operator quantisation in loop quantum gravity I, II Class. Quantum Grav. 23 5667-772

[28] Koslowski T 2007 private communication

[29] Rourke C P and Sanderson B J 1982 Introduction to Linear Topology (Berlin: Springer) 